\title{
X-ray emission from NGC 1808: more than a complex starburst ${ }^{\star}$
}

\author{
E. Jiménez-Bailón ${ }^{1}$, M. Santos-Lleó ${ }^{1}$, M. Dahlem ${ }^{2}$, M. Ehle ${ }^{1}$, J. M. Mas-Hesse ${ }^{3}$, M. Guainazzi ${ }^{1}$, \\ T. M. Heckman ${ }^{4}$, and K. A. Weaver ${ }^{5}$
}

\author{
1 XMM-Newton Science Operations Centre, ESAC, ESA, Apartado 50727, 28080 Madrid, Spain \\ e-mail: Elena.Jimenez@sciops.esa.int \\ 2 ATNF/CSIRO, Paul Wild Observatory, Locked Bag 194, Narrabri NSW 2390, Australia \\ 3 Centro de Astrobiología (CSIC-INTA), 28850 Torrejón de Ardoz, Madrid, Spain \\ ${ }^{4}$ Dept. of Physics and Astronomy, Johns Hopkins University, 3400 N. Charles St., Baltimore, MD 21218, USA \\ 5 Laboratory for High Energy Astrophysics, NASA's Goddard Space Flight Center, Greenbelt, MD 20771, USA
}

Received 8 March 2005 / Accepted 10 May 2005

\section{ABSTRACT}

Earlier observations of NGC 1808 in various wavebands (X-ray, optical, near-infrared, radio) provided evidence for the existence of either a starburst or a Seyfert 2 nucleus. We here present the results of multiwavelength XMM-Newton and Chandra observations, which directly prove the co-existence of thermal diffuse plasma and non-nuclear unresolved point-like sources associated with the starburst activity, along with a Low Luminosity Active Galactic Nucleus (LLAGN) or an Ultra Luminous X-ray source (ULX). The broad bandwidth of XMM-Newton allows us to show that the unresolved nuclear source in NGC 1808 dominates the hard X-ray spectrum, while the emission in the soft regime, below 1 $\mathrm{keV}$, is dominated by a thermal component associated to an extended starburst. Both EPIC and RGS data provide reliable detections of a number of emission lines from heavy elements, with abundances ranging from roughly 0.7 to $2.2 Z_{\odot}$ for different elements. However, no $6.4 \mathrm{keV}$ Fe $\mathrm{K} \alpha$ fluorescence line emission was detected. The analysis of the nuclear region of NGC 1808 allows us to detect and disentangle the contribution of an unresolved nuclear X-ray source and the starburst region, but the exact nature of the nucleus remains unknown. The observed luminosity of NGC 1808 is $L_{2-10 \mathrm{keV}}=(1.61 \pm 0.06) \times 10^{40} \mathrm{erg} \mathrm{s}^{-1}$. A comparison of our OM $212 \mathrm{~nm}$ image with a CTIO 4-m telescope H $\alpha$ frame shows a good general correspondence between the emission from massive stars and warm ionized gas, with minor deviations near the ends of the bar in NGC 1808. An aditional, very soft thermal spectral component with $k T \simeq 0.1 \mathrm{keV}$ has been discovered in the XMM-Newton spectral analysis, which most likely originates from the halo of NGC 1808.

Key words. galaxies: active - galaxies: nuclei - galaxies: general - X-rays: general - galaxies: evolution galaxies: halos - galaxies: starburst - individual: NGC 1808

\section{Introduction}

NGC $1808 \quad(\alpha(\mathbf{J} 2000)=05: 07: 42.34 ; \quad \delta(\mathbf{J} 2000)=-37: 30: 47.0$ with an uncertainty of 1. '.25, obtained from $2 \mathrm{MASS}^{1}$ ), classified as an SABb Seyfert 2 galaxy, is located at a distance of $10.9 \mathrm{Mpc}\left(H_{0}=75 \mathrm{~km} \mathrm{~s}^{-1} \mathrm{Mpc}^{-1}, 1^{\prime \prime}=53 \mathrm{pc}\right)$. Images in different wavebands suggest a high star formation (SF) intensity in the central region with a diameter of $\sim 20^{\prime \prime}$ (i.e., $\approx 1 \mathrm{kpc}$ ). There are several optical hot spots associated with $\mathrm{H}$ II-regions (Sérsic \& Pastoriza 1965). The luminous and compact knots detected in radio and infra-red (IR) images (Saikia et al. 1990; Kotilainen et al. 1996) that do not coincide with the optical hot spots are probably supernova remnants (SNRs) or complexes of unresolved SNRs. Dust filaments were found perpendicular to

^ Partially based on observations obtained with XMM-Newton, an ESA science mission with instruments and contributions directly funded by ESA Member States and NASA.

1 http://www.ipac.caltech.edu/2mass/ the central disk plane and can be explained as outflowing material driven by supernovae ( $\mathrm{SNe}$; Heckman et al. 1990). A recent interaction of NGC 1808 with its companion NGC 1792 could explain both the intense star-formation activity and its peculiar morphology (Dahlem et al. 1990; Koribalski et al. 1993).

The nature of the nucleus is still unclear. It is classified as Seyfert 2 based on the optical emission lines detected by Véron-Cetty \& Véron (1985). However, there is also evidence from optical observations of a hidden starburst (SB), based on polarization measurements by Scarrott et al. (1993). The nucleus is claimed to have a strong non-stellar component, because only $10 \%$ of the IR radiation observed by ISO could be interpreted as emission related to star formation (Siebenmorgen et al. 2001). On the other hand, soft X-ray $(0.1-2.4 \mathrm{keV})$ data obtained with ROSAT (Dahlem et al. 1994; Junkes et al. 1995) favor an interpretation in terms of SF-related emission, but do not discard other hypotheses. Awaki \& Koyama (1993) interpret their Ginga X-ray data (1.5-37 keV) as an obscured active 
galactic nucleus (AGN). However, Awaki et al. (1996), using ASCA (2-10 keV) observations, point out that the hard X-ray spectrum could also be the result of starburst activity, while the long-term variability from the Ginga and ASCA observations again suggests a Seyfert nature of the nucleus.

With the advent of the XMM-Newton and Chandra satellites, the coexistence of starburst and AGN activity has been detected in X-rays in the nuclei of several nearby galaxies. NGC 4303 presents evidence of the composite SB/AGN nature of the nuclear region: Chandra and UV-HST observations show that a superstellar cluster coexists with a hard X-ray source, possibly an AGN, in the 3 central pc of the galaxy (Jiménez-Bailón et al. 2003; Colina et al. 2002). At larger scales, Persic et al. (2004a) claim that starburst and AGN coexist in NGC 4666 by detecting evidence of diffuse thermal emission, high-luminousity X-ray sources, and the presence of an AGN in the X-ray band. The detailed analysis of our $X M M-N e w t o n$ data shown here, combined with other observations, sheds new light on the nature of the nuclear activity in NGC 1808 and the associated outflow of gas.

The observations and data reduction are described in Sect. 2, our results are presented in Sect. 3, followed by our interpretation of the inner region and circumnuclear emission in Sect. 4.1 and of the more extended X-ray emission outside the nuclear area in Sect. 4.2. The conclusions of this work are given in Sect. 5.

\section{Observations and data reduction}

\subsection{XMM-Newton data}

Information on the XMM-Newton instrumentation is provided by Jansen et al. (2001; XMM-Newton mission), Strüder et al. (2001; EPIC-pn), Turner et al. (2001; EPIC-MOS), den Herder et al. (2001; RGS), and Mason et al. (2001; OM). Our XMM-Newton observation (Obs-Id 0110980801) was performed on April 6, 2002. The scheduled exposure time was $40 \mathrm{ks}$, while effective exposure times per instrument are listed below. The EPIC-pn exposure was in extended full frame mode and with the thin filter. Both $R G S$ cameras were in spectroscopic mode and the two MOS cameras in full frame, with the thin filter. $O M$ was used in full frame low resolution mode with the U, UVW1 $(219 \mathrm{~nm})$ and UVW2 $(212 \mathrm{~nm})$ broad band filters, plus the two (optical and UV) grisms.

The data were processed with the Science Analysis Subsystem, SAS, v.5.4.1 (Gabriel et al. 2004) and the most upto-date calibration available in February 2003. The standard tasks to process data, ep/mchain and rgsproc, were run with default parameters. For the EPIC instruments, checks against pile-up were performed with the epatplot task. No sign of pile-up was found for any of EPIC-pn, MOS1, or MOS2 data. Additionally, background flaring time intervals, for which the signal-to-noise does not improve by including these events, were filtered following the method described in Piconcelli et al. (?b). Only single and double events were considered in the analysis. For $R G S$ the selection was for periods with less than $0.12 \mathrm{c} / \mathrm{s}$ in the background region of the CCD number 9 . The effective exposure times after each filter was applied were
$31.4 \mathrm{ks}, 39.0 \mathrm{ks}, 38.6 \mathrm{ks}$, and $37.4 \mathrm{ks}$ for EPIC-pn, the two MOS cameras, and $R G S$, respectively. The $O M$ broad band data were processed with the SAS omichain task, which performed all necessary corrections. Unfortunately, about half of the UVW1 filter image was lost due to ground-station problems, and the UV grism data was also lost due to telemetry drops.

\subsection{Chandra data}

The Chandra ACIS (AXAF CCD Imaging Camera) archival image, sequence number 700451, was extracted from the Chandra archive. The ACIS observation took place on December 19, 2002 with an exposure time of $43 \mathrm{ks}$. We used the science level 2 files, generated by the pipeline standard processing on December 19, 2002, as stored in the archive. They were processed with Ciao 3.0.2. According to the information available on the Chandra pages, further corrections should be applied (i.e. reduction of tap-ringing distortions). However, for the purpose of the present work, this was found to be unnecessary. No additional corrections were therefore applied.

\subsection{Ground-based $\mathrm{H} \alpha$ imagery}

The $\mathrm{H} \alpha$ data used here were obtained with the CTIO 4-m telescope on November 18, 1993. A $2048 \times 2048$ chip with a 0!' 431 pixel scale was used, leading to a total field-of-view of $14.7 \times 14.7$.

Three exposures of $20 \mathrm{~min}$. each were taken with a redshifted $\mathrm{H} \alpha$ filter $\left(\lambda_{0}=657.8 \mathrm{~nm} ; \Delta \lambda=0.64 \mathrm{~nm}\right)$, with a total integration time of $1 \mathrm{hr}$. The continuum subtraction was performed using an $R$-band image with a total integration time of $8 \mathrm{~min}$. The data are extremely sensitive to low surface brightness emission, but as they are uncalibrated, will be used only for qualitative studies.

\section{Results}

\subsection{X-ray imaging}

XMM-Newton EPIC X-ray images of NGC 1808 were produced by grouping the photons from the cleaned event lists into the sub-bands defined by the XMM-Newton Survey Science Centre (SSC; Watson et al. 2001) and using the merge task in SAS v.5.4.1, which takes exposure time corrections into account. These images are displayed in Fig. 1. The maximum emission is located at $\alpha^{\mathrm{EPIC}}(\mathrm{J} 2000)=05: 07: 42.40$; $\delta^{\mathrm{EPIC}}(\mathrm{J} 2000)=-37: 30: 46.2$ with an uncertainty of $11^{\prime \prime} 5-3^{\prime \prime}$. The position of the 2MASS nucleus is indicated by a cross. An adaptive filter was used to smooth the images in areas of low signal-to-noise $(\mathrm{S} / \mathrm{N})$ ratio, while not smearing out the emission from point sources.

NGC 1808 shows extended emission in the soft X-ray images up to $4.5 \mathrm{keV}$ (Fig. 1). Figure 2 shows the comparison of the radial profiles of NGC 1808 and MCG-06-3015 in two energy bands: $0.2-4.5 \mathrm{keV}$ and $4.5-10 \mathrm{keV}$. The QSO MCG-06-30-15 can be considered as a point-like source in all the XMM-Newton energy bands (Kirsch et al. 2005). The comparison shows that the emission of NGC 1808 in the 


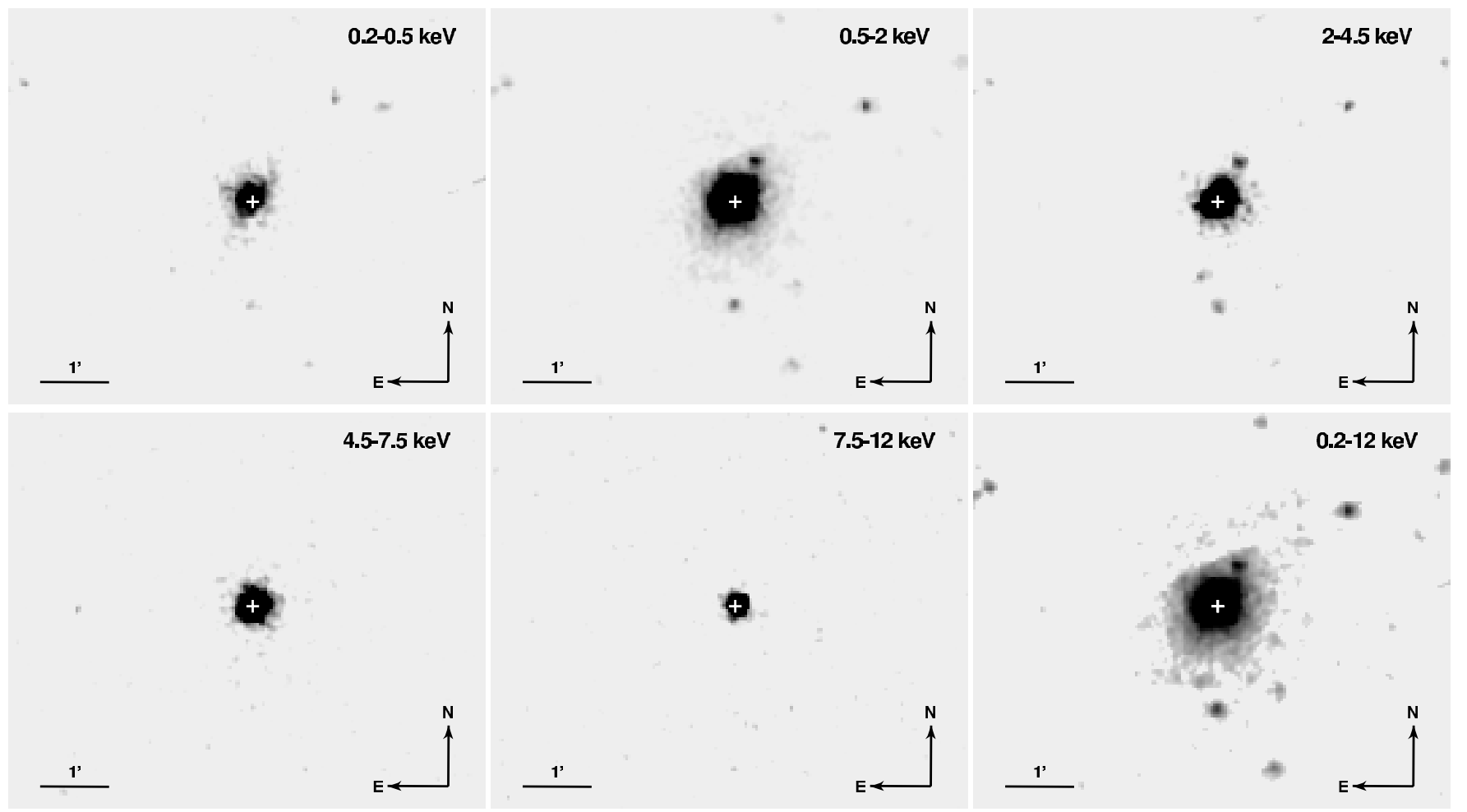

Fig. 1. EPIC images of NGC 1808 in the following energy bands (from left to right and from top to bottom): $0.2-0.5 \mathrm{keV}, 0.5-2.0 \mathrm{keV}$, $2.0-4.5 \mathrm{keV}, 4.5-7.5 \mathrm{keV}, 7.5-12 \mathrm{keV}$, and the total bandpass $0.2-12 \mathrm{keV}$. All images are displayed on a logarithmic scale with a threshold of $3 \sigma$ of the mean background value.

lowest energy band is extended while its radial profile in the 4.5-10 keV band does not differ from the QSO profile, taking the errors into account. Therefore, NGC 1808 can be considered as a point-like source above $4.5 \mathrm{keV}$. This points out that the origin of the X-ray emission is not due to the compact central source alone. The elongated X-ray emission of NGC 1808 follows the same orientation as the $\mathrm{H} \alpha$ emission (see Sect. 3.4). The extended morphology is detected at energies below $4.5 \mathrm{keV}$, with a maximum in the $0.5-2.0 \mathrm{keV}$ band (see Fig. 1). At these low energies, thermal emission processes tracing high-mass SF play an important role. Thus, our data suggest that the stellar activity in the nuclear region is significantly contributing to the observed soft X-ray emission. With increasing energy, the central emission maximum becomes more prominent and the non-nuclear sources fade away.

In order to resolve more details, we have compared our 0.2-12 keV EPIC image with the Chandra ACIS data. Both were smoothed with an adaptive filter and are displayed in Fig. 3. The Chandra ACIS image shows how the nucleus is surrounded by a population of discrete sources. The image (left panel of Fig. 3) shows the detailed structure in the central part of NGC 1808: at least four point-like sources are detected in the image, apart from the diffuse emission. Two of them are located at $\sim 4^{\prime \prime}(212 \mathrm{pc}) \mathrm{NW}$ and SE from the maximum of the X-ray emission in the Chandra image and have also been detected in a Chandra HRC observation. Zezas et al. (2001) reported a luminosity for each of them of around $10^{39} \mathrm{erg} \mathrm{s}^{-1}$.

Although the nucleus is observed as a point-like source in the HRC image (see Zezas el al. 2001), the ACIS image reveals the presence of a double-peak structure (see Fig. 3). The hard $(E>2 \mathrm{keV})$ and soft $(E<1.5 \mathrm{keV}) \mathrm{X}$-ray images of the two peaks presented in Fig. 4 reveal that the eastern source, $\mathrm{S} 1$, is significantly softer than the western one, S2. The locations of the two peaks are $\alpha_{1}^{\text {Chandra }}(\mathrm{J} 2000)=05: 07: 42.35$; $\delta_{1}^{\text {Chandra }}(\mathbf{J} 2000)=-37: 30: 45.8 \quad$ and $\quad \alpha_{2}^{\text {Chandra }}(\mathbf{J} 2000)=$ $05: 07: 42.19 ; \quad \delta_{2}^{\text {Chandra }}(\mathbf{J} 2000)=-37: 30: 45.8$ with an uncertainty of 0.' 6 . The nuclear location from 2MASS is indicated by an arrow in the Chandra image, closer to the eastern source found by Chandra, S1, but compatible within the errors with both of them. The remaining two point-like sources are situated on the ring of radio emission as imaged by Saikia et al. (1990), close to the detected SNR and H II-regions.

\subsection{X-ray spectral analysis}

We performed an extensive X-ray spectral analysis of NGC 1808 using the EPIC and RGS instruments on board $X M M-N e w t o n$, as well as, with Chandra's ACIS camera.

The XMM-Newton EPIC data were used to perform a spectral analysis of NGC 1808 in the $0.35-9 \mathrm{keV}$ energy band with moderate resolution $(80 \mathrm{eV}$ at $1 \mathrm{keV})$. In order to search for differences in the X-ray spectrum of the nucleus and its surroundings, we defined three circular regions and one annulus. The circles have radii of $16^{\prime \prime}(850 \mathrm{pc}), 35^{\prime \prime}(1.9 \mathrm{kpc})$, and $2^{\prime}(6.4 \mathrm{kpc})$, while the annulus has external and internal radii of $20^{\prime \prime}$ and $50^{\prime \prime}(1.0-2.7 \mathrm{kpc})$, respectively (excluding one extra-nuclear source). Background regions were selected to be on the same CCD as the source but far enough to prevent source 

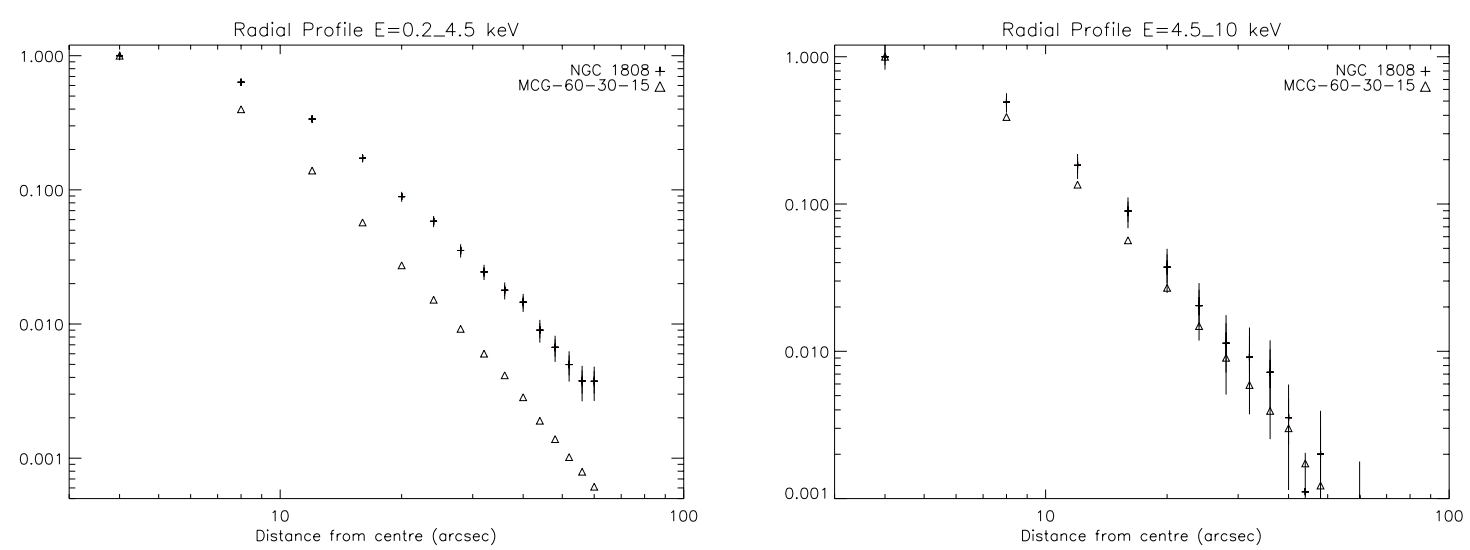

Fig. 2. Comparison between the radial profiles of NGC 1808 and the point-like source MCG-06-30-15 in the $0.2-4.5 \mathrm{keV}$ and $4.5-10 \mathrm{keV}$ bands. $Y$-axis shows the normalised brightness (normalised counts $/ \mathrm{s} / \mathrm{px}^{2}$ ). The plots show that the emission of NGC 1808 is extended in the $0.2-4.5 \mathrm{keV}$ band and point-like in the $4.5-10 \mathrm{keV}$ band.
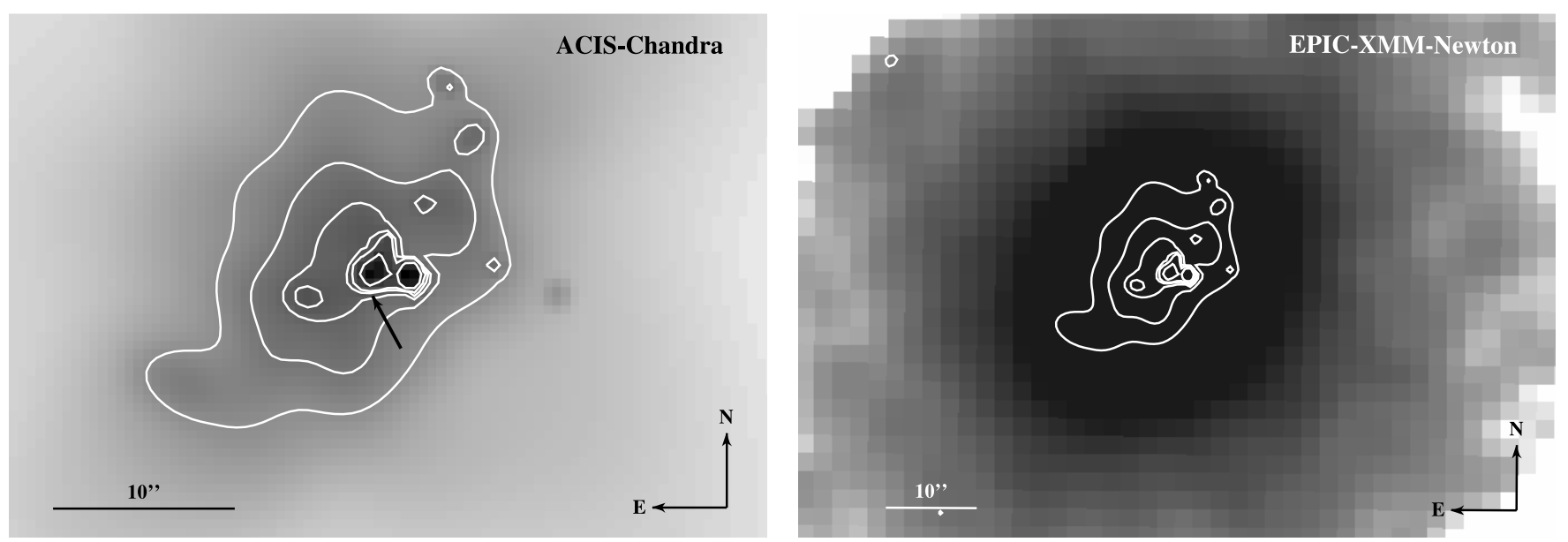

Fig. 3. NGC 1808 0.2-12 keV X-ray images taken with the Chandra ACIS (left panel) and the XMM-Newton EPIC (right panel) with the ACIS image overlaid as contours (contour levels are 2, 5, 10, 15, 20, and 50 counts). The nuclear location from 2MASS is indicated by an arrow in the Chandra image. Both images are displayed on a logarithmic scale with a threshold of $3 \sigma$ of the mean background value.

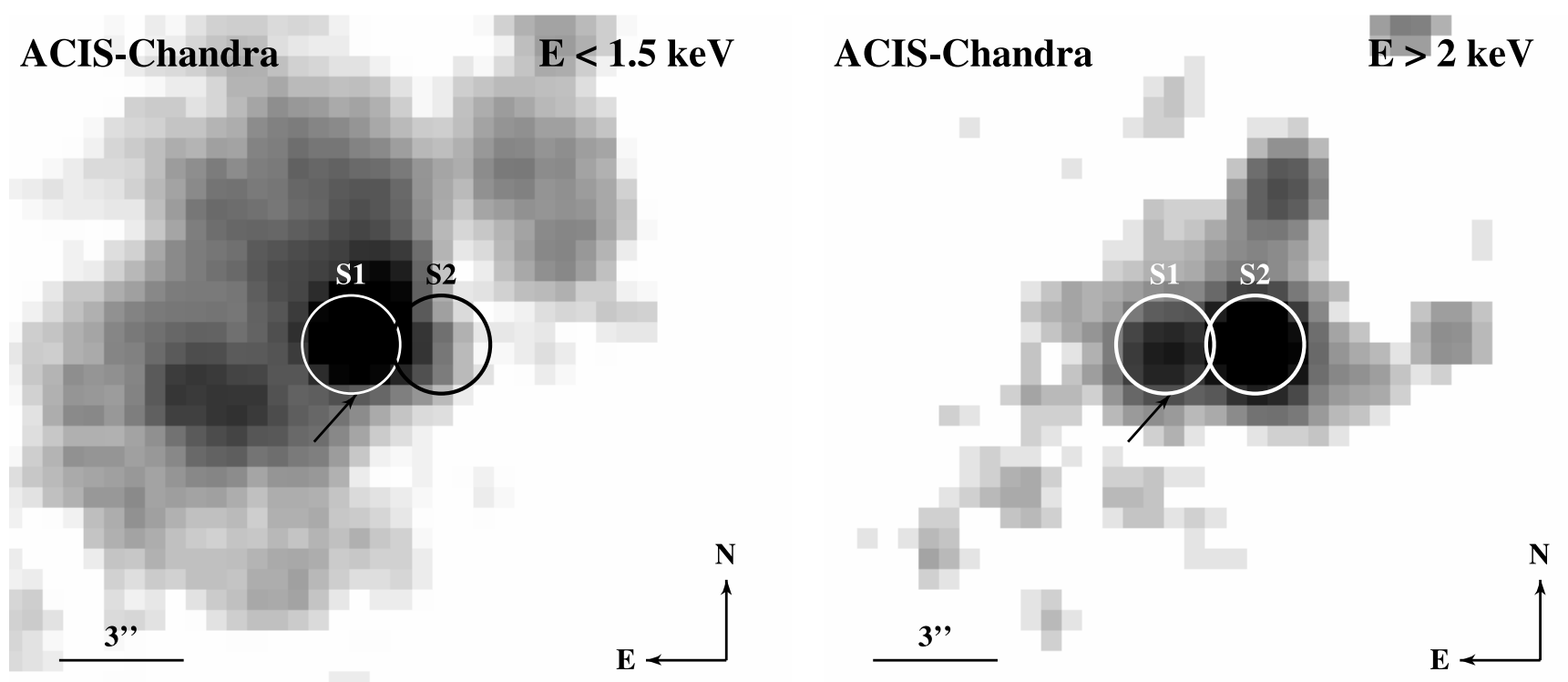

Fig. 4. The Chandra ACIS hard $(E>2 \mathrm{keV})$ and $\operatorname{soft}(E<1.5 \mathrm{keV}) \mathrm{X}$-ray images of the two nuclear point-like sources. The eastern source, S1, is softer than the western one, S2. The nuclear location from 2MASS, indicated by an arrow in the images, is closer to source S1. The circles indicate the regions used for spectral extraction (Sect. 3.2.2). 


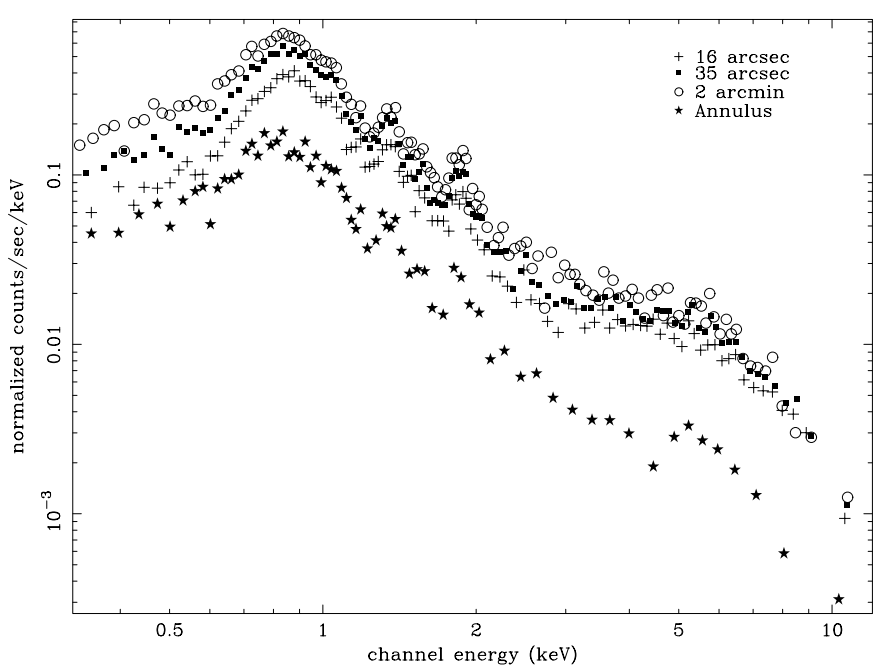

Fig. 5. EPIC spectra of the three circular regions and the annulus (see text for further details).

contamination. Figure 5 shows the spectra of each region. We simultaneously analysed the EPIC-pn and RGS spectra, and finally we also studied the Chandra spectra of the two nuclear point-like sources S1 and S2 (see Fig. 4), both located within the $16^{\prime \prime}$ inner region extracted in the XMM-Newton analysis.

In order to apply the modified $\chi^{2}$ minimization technique in the spectral analysis, all EPIC spectra were grouped such that each spectral bin contains at least 50 counts. The Chandra spectra were grouped with at least 20 counts in each bin. The spectra were analysed using XSPEC v.11.3.0 (Arnaud 1996).

The spectra of the $35^{\prime \prime}$ and $2^{\prime}$ regions are very similar, both in shape and intensity (see Fig. 5). Only the spectrum of the 16" region, although in good agreement with the two previous ones above $2 \mathrm{keV}$, is clearly weaker in the soft band.

\subsubsection{EPIC spectrum of the inner region}

The EPIC spectrum of the inner circular region of NGC 1808 is displayed in Fig. 6. The extraction region has a radius of $16^{\prime \prime}$ $(850 \mathrm{pc})$, centred on the maximum peak of the X-ray emission. MOS1 and MOS2 spectra and response matrices were combined by merging these to maximize the signal-to-noise level. Subsequently, the EPIC-pn and combined MOS spectra were fitted simultaneously. Figure 6 shows the $0.35-9 \mathrm{keV}$ spectrum, the best fit model with a statistical quality of $\chi_{v}^{2}=0.99$ for 174 degrees of freedom (d.o.f.), and the residuals.

Simple models as a power law, a Raymond-Smith (Raymond \& Smith 1976), mekal (Mewe et al. 1986), and bremsstrahlung components provide unacceptable fits. The best fit model includes, apart from the fixed Galactic foreground absorption with a column density of $N_{\mathrm{H}}=3.23 \times 10^{20} \mathrm{~cm}^{-2}$ (Dickey \& Lockman 1990), a power law with index $\Gamma=$ $0.79_{-0.06}^{+0.07}$ and a mekal thermal component with a temperature of $k T=0.580_{-0.016}^{+0.019} \mathrm{keV}$ absorbed by a Hydrogen column of $N_{\mathrm{H}}=2.0_{-0.4}^{+0.3} \times 10^{21} \mathrm{~cm}^{-2}$. The value of $\chi^{2}$ is unacceptable, but an intrinsic absorption to the power law component does not improve the fit; the inferred upper limit for the Hydrogen column density is $8 \times 10^{20} \mathrm{~cm}^{-2}$. The fit only improved, in terms

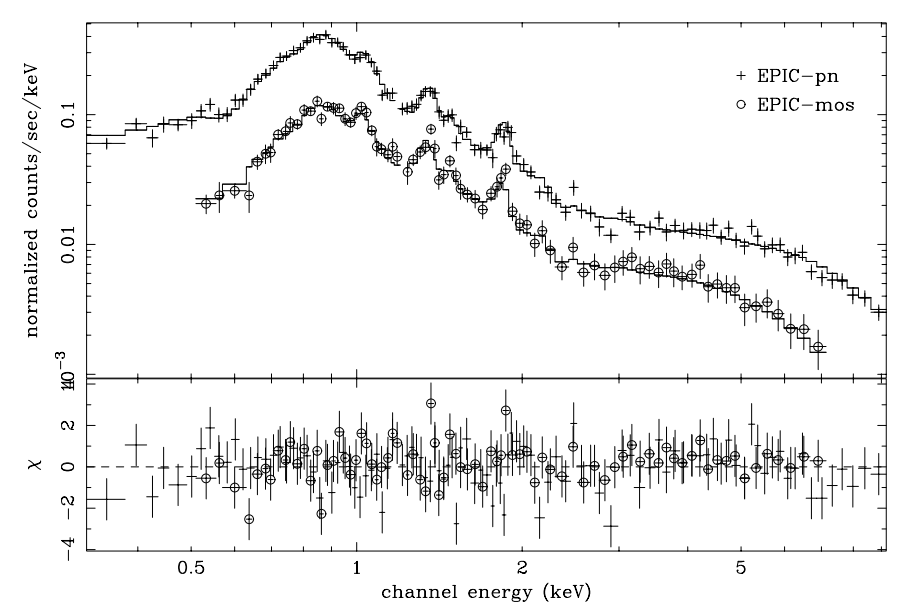

Fig. 6. EPIC spectrum, best fit model, and residuals of the $r=16^{\prime \prime}$ inner region.

of $\chi^{2}$, when some abundances were allowed to differ from the solar values. The abundances of $\mathrm{Ne}, \mathrm{Mg}, \mathrm{Si}$, and $\mathrm{Fe}$, left free in the fits, are $1.6_{-0.5}^{+1.0}, 2.2_{-0.5}^{+1.2}, 2.1_{-0.5}^{+1.0}$, and $0.63_{-0.11}^{+0.2}$ times the solar value $\left(Z_{\odot}\right)$, respectively. Fits with other thermal models such as Raymond-Smith or bremsstrahlung emission lead to higher values of $\chi^{2}$. No Fe $\mathrm{K} \alpha$ line was significantly detected. An upper limit of $80 \mathrm{eV}$ was calculated for the equivalent width of a narrow iron line. Table 1 shows the goodness of the fit together with the values and uncertainties for each free parameter.

\subsubsection{The Chandra spectra of sources $\mathrm{S} 1$ and $\mathrm{S} 2$}

The ACIS image in Fig. 3 shows a double-peak nuclear structure surrounded by a few point-like sources inside the inner, $r=16^{\prime \prime}$, region defined for EPIC and analysed in the previous sub-section. We analysed the standard extracted spectra obtained with psextract of the two circular, $r=1^{\prime \prime} 2$, regions S1 and S2 (see Fig. 4). The background was extracted from a clean circular region with a radius of $10^{\prime \prime}$ located $\sim 1^{\prime}$ North-East from the sources in the same CCD. For the two regions, models with a single component were rejected due to the high $\chi^{2}$ value obtained.

The best fit model obtained for the observed spectrum of the hard source, S2, includes an absorbed mekal component and an absorbed power law. The values of the parameters and the goodness of the fit are presented in Table 2 and the observed data, the best fit model, and the residuals are shown in Fig. 7b. It is worth noting the high values of the absorbing column densities for both components, $N_{\mathrm{H}}=(2-3) \times 10^{22} \mathrm{~cm}^{-2}$. The index of the power law found is $\Gamma=1.2 \pm 0.3$, and the temperature of the thermal component is $k T=0.087_{-0.006}^{+0.005} \mathrm{keV}$. The thermal component in S2 is significantly colder than the one detected in the $E P I C$ spectrum of the inner region, with $k T=0.58 \pm 0.02 \mathrm{keV}$. The fit does not improve by adding an extra thermal component that could account for the thermal emission observed in the inner region of XMM-Newton, $k T=0.58 \mathrm{keV}$. Neither does it improve by varying the abundances from the solar value. 
Table 1. Parameters and goodness of the best fit models for the $r=16^{\prime \prime}, r=62$.' 5 circular regions, and the $16^{\prime \prime} 5-35^{\prime \prime}$ annular region.

\begin{tabular}{|c|c|c|c|c|c|c|c|c|}
\hline Region & $\begin{array}{l}N_{\mathrm{H}} \\
10^{21} \mathrm{~cm}^{-2}\end{array}$ & $\Gamma$ & $\begin{array}{l}A_{\mathrm{pl}}^{\dagger} \\
10^{-5}\end{array}$ & $\begin{array}{l}k T_{\mathrm{I}} \\
\mathrm{keV}\end{array}$ & $\begin{array}{l}A^{\ddagger} \\
10^{-4}\end{array}$ & $\begin{array}{l}k T_{\text {II }} \\
\mathrm{keV}\end{array}$ & $\begin{array}{l}A^{\ddagger} \text { II } \\
10^{-4}\end{array}$ & Abundances \\
\hline$\chi^{2}=172$ for 174 d.o.f. & $2.0_{-0.4}^{+0.3}$ & $0.79_{-0.06}^{+0.07}$ & $6.00_{-0.6}^{+0.7}$ & $0.580_{-0.016}^{+0.019}$ & $3.7_{-1.2}^{+0.9}$ & - & - & $\begin{array}{l}\text { Ne } 1.6_{-0.5}^{+1.0} \\
\text { Mg } 2.2_{-0.5}^{+1.2} \\
\text { Si } 2.1_{-0.5}^{+1.0} \\
\text { Fe } 0.63_{-0.11}^{+0.2}\end{array}$ \\
\hline$\chi^{2}=135$ for 88 d.o.f. & 2.0 (f) & $0.79(\mathrm{f})$ & $0.93 \pm 0.08$ & 0.58 (f) & $1.1 \pm 0.4$ & $0.096_{-0.008}^{+0.015}$ & $2.5_{-1.5}^{+5}$ & $\begin{array}{l}\text { Ne } 0.6_{-0.3}^{+0.4} \\
\text { Mg } 1.0_{-0.4}^{+0.5} \\
\text { Si } 1.2_{-0.4}^{+0.5} \\
\text { Fe } 0.37_{-0.04}^{+0.12}\end{array}$ \\
\hline $\begin{array}{l}62^{\prime \prime} .5(3.3 \mathrm{kpc}) \\
\chi^{2}=222 \text { for } 222 \text { d.o.f. }\end{array}$ & $1.87 \pm 0.03$ & $0.79(\mathrm{f})$ & $6.5 \pm 0.2$ & $0.572_{-0.04}^{+0.018}$ & $4.8_{-1.1}^{+0.7}$ & $0.11_{-0.02}^{+0.03}$ & $4_{-3}^{+15}$ & $\begin{array}{l}\mathrm{Ne} 1.5_{-0.4}^{+0.5} \\
\mathrm{Mg} 1.9_{-0.4}^{+0.6} \\
\text { Si } 1.9_{-0.5}^{+0.7} \\
\text { Fe } 0.51_{-0.08}^{+0.11}\end{array}$ \\
\hline
\end{tabular}

The best model for $16^{\prime \prime}$ region is phabs $\mathrm{Gal}_{\mathrm{G}}\left(\mathrm{zpow}+\mathrm{zphabs}{ }^{*} \mathrm{vmekal}\right)$ and the best fit model for $16^{\prime \prime} 5-35^{\prime \prime}$ and $62^{\prime \prime} 5$ regions is phab $_{\mathrm{Gal}}\left(\right.$ zpow+zphabs* $\left(\operatorname{vmekal}_{\mathrm{I}}+\operatorname{vmekal}_{\mathrm{II}}\right)$. Normalisation in units of: $\left(^{\dagger}\right)$ photons $/ \mathrm{keV} / \mathrm{cm}^{2} / \mathrm{s}$ at $1 \mathrm{keV}$ and $\left(^{(}\right)$in $\frac{10^{-14}}{4 \pi D^{2}(1+z)^{2}} \int n_{\mathrm{e}} n_{\mathrm{H}} \mathrm{d} V$.

Table 2. Parameters and goodness of the best fit models for sources S1 and S2 observed with Chandra.

\begin{tabular}{|c|c|c|c|c|c|c|c|}
\hline Region & $\begin{array}{l}N_{\mathrm{H}}(\mathrm{pwlw}) \\
10^{22} \mathrm{~cm}^{-2}\end{array}$ & $\begin{array}{l}N_{\mathrm{H}}(\text { mekal }) \\
10^{22} \mathrm{~cm}^{-2}\end{array}$ & $\bar{\Gamma}$ & $\begin{array}{c}A_{\mathrm{pl}}^{\dagger} \\
10^{-5}\end{array}$ & $\begin{array}{l}k T \\
\mathrm{keV}\end{array}$ & $A^{\ddagger}$ & Abundances \\
\hline Source S1 & - & $0.52_{-0.07}^{+0.10}$ & $0.99_{-0.14}^{+0.18}$ & $0.7 \pm 0.4$ & $0.58_{-0.09}^{+0.07}$ & $9_{-2}^{+3} \times 10^{-5}$ & $\begin{array}{l}\mathrm{Ne} 1.6(\mathrm{f}) \\
\mathrm{Mg} 2.2(\mathrm{f}) \\
\mathrm{Si} 2.2(\mathrm{f})\end{array}$ \\
\hline$\chi^{2}=30$ for 25 d.o.f. & & & & & & & $\mathrm{Fe} 0.63(\mathrm{f})$ \\
\hline $\begin{array}{l}\text { Source } \mathbf{S 2} \\
\chi^{2}=41 \text { for } 28 \text { d.o.f. }\end{array}$ & $3.1_{-0.7}^{+0.8}$ & $2.0_{-0.4}^{+0.3}$ & $1.2 \pm 0.3$ & $6_{-3}^{+7}$ & $0.087_{-0.006}^{+0.005}$ & $2_{-2}^{+6}$ & - \\
\hline
\end{tabular}

The best models for regions S1 and S2 are phabs ${ }_{\mathrm{Gal}}\left(\mathrm{zpow}+\mathrm{zphabs}_{\mathrm{mekal}} * \mathrm{vmekal}\right)$ and phabs $\mathrm{Gal}_{\mathrm{Gal}}\left(\mathrm{zphabs}_{\mathrm{pwlw}}{ }_{\mathrm{zpow}+\mathrm{zphabs}} \mathrm{mekal} * \mathrm{mekal}\right)$, respectively. Normalisation in units of: $\left(^{\dagger}\right)$ photons $/ \mathrm{keV} / \mathrm{cm}^{2} / \mathrm{s}$ at $1 \mathrm{keV}$ and $\left(^{\ddagger}\right)$ in $\frac{10^{-14}}{4 \pi D^{2}(1+z)^{2}} \int n_{\mathrm{e}} n_{\mathrm{H}} \mathrm{d} V$.
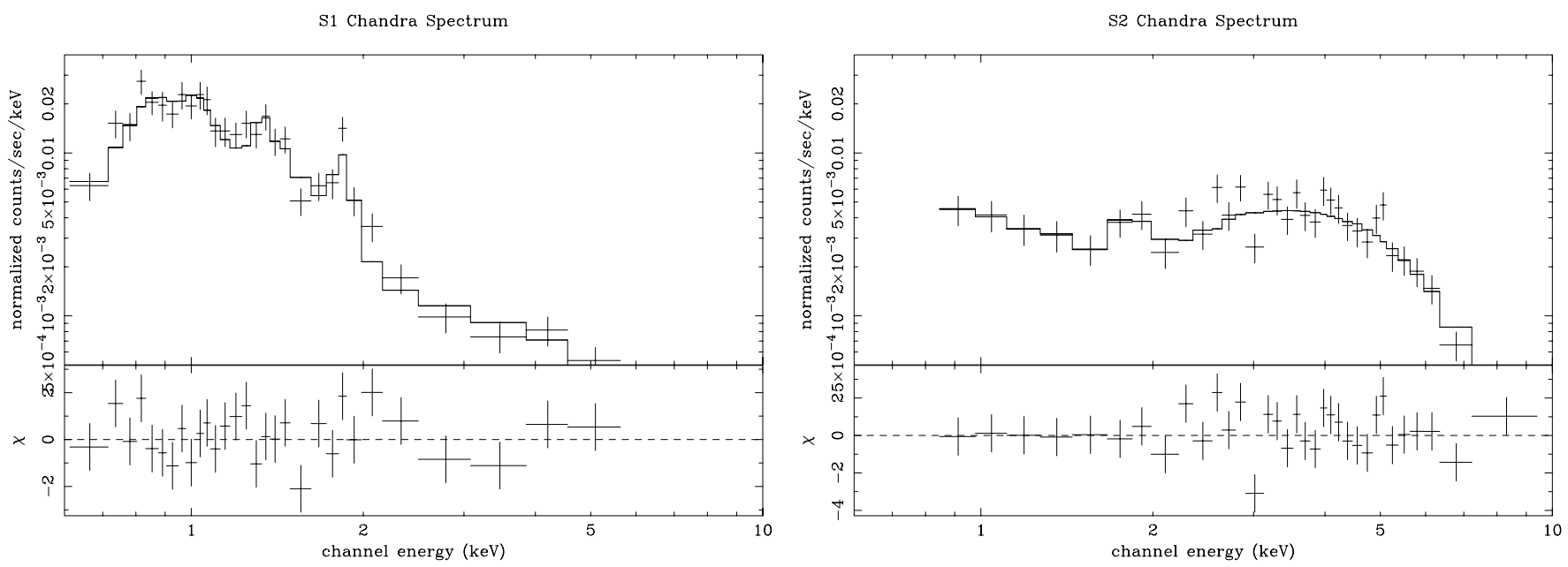

Fig. 7. Chandra spectra of source $\mathrm{S} 1$ a) and source $\mathrm{S} 2 \mathbf{b}$ ), the corresponding best fit models, and the residuals. Note that the $y$-axis scale is the same on both plots to allow comparison of the spectra of both sources.

Figure 4 shows that above $2 \mathrm{keV}$, source $\mathrm{S} 1$ is dimmer than source S2, while below $1.5 \mathrm{keV}$ it is the opposite. Assuming that the low hard emission of $\mathrm{S} 1$ could be due to contamination of the harder source, S2, we tested a model which includes an absorbed power law component with photon index and $N_{\mathrm{H}}$ fixed to the values obtained for the best fit model of source S2. In order to model the soft range, we considered an absorbed mekal thermal model with free temperature. The resulting fit is unacceptable, $\chi_{v}^{2}=1.9$. The addition of an extra thermal component with $k T=0.087 \mathrm{keV}$ equal to the one detected in source 

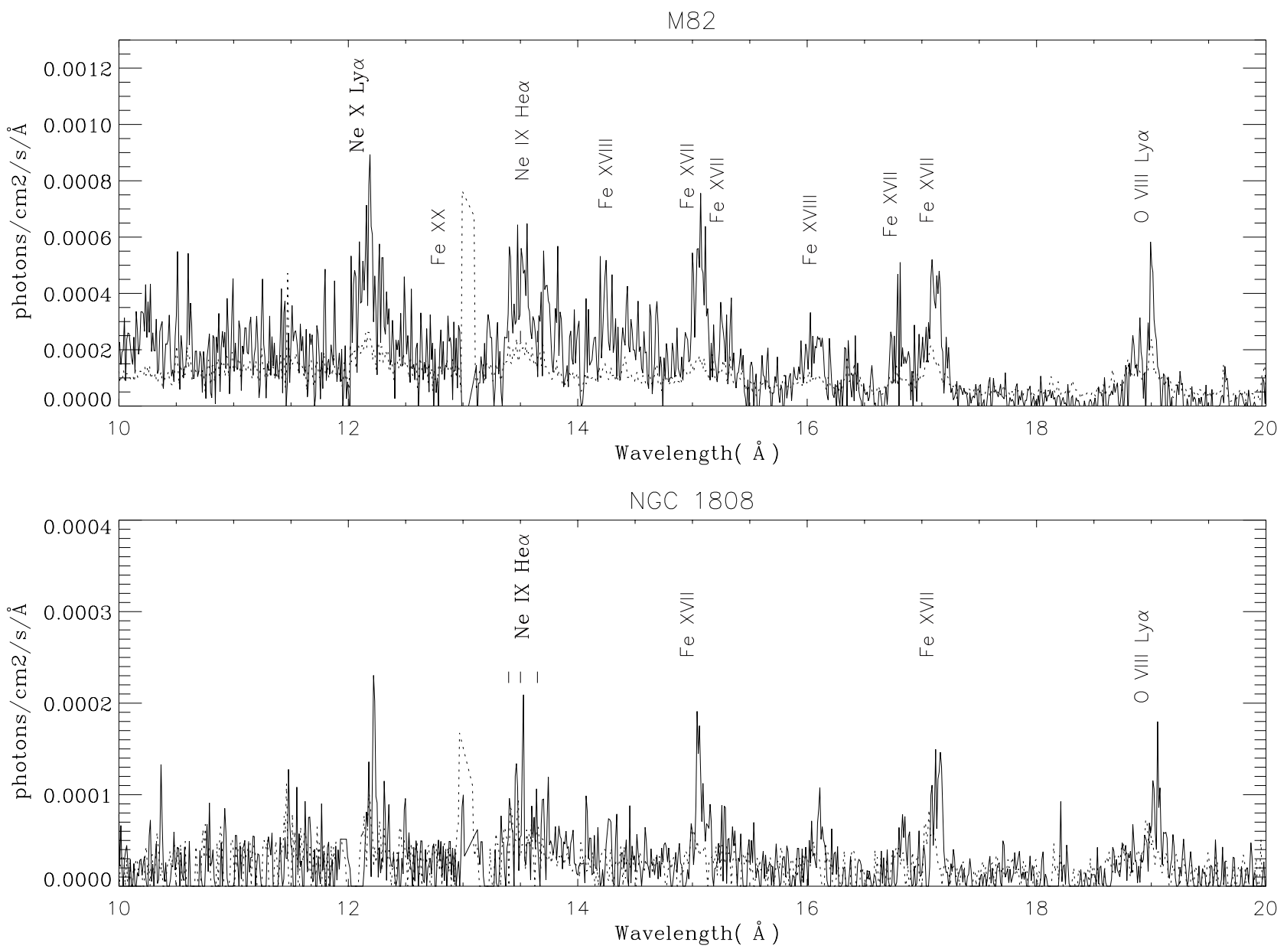

Fig. 8. RGS fluxed spectra of M 82 and NGC 1808, upper and lower panel, respectively. The dotted line corresponds to the errors. The identification of M 82 lines is from Reads \& Stevens (2002).

S2 does not significantly improve the fit. The best fit model is obtained with an unabsorbed power law with $\Gamma=0.99_{-0.14}^{+0.18}$ and a moderately absorbed, $N_{\mathrm{H}}=5.2_{-0.7}^{+1.0} \times 10^{21} \mathrm{~cm}^{-2}$, mekal component with a temperature of $k T=0.58_{-0.09}^{+0.07} \mathrm{keV}$ and the abundances of $\mathrm{Ne}, \mathrm{Mg}, \mathrm{Si}$, and $\mathrm{Fe}$ fixed to the values obtained in the fit of the EPIC spectrum of the inner region. If an absorption component above the Galactic value is applied to the power law, the value of $N_{\mathrm{H}}$ for that additional component is compatible with zero. The observed data, the best fit model, and the residuals are shown in Fig. 7a.

\subsubsection{Simultaneous analysis of EPIC-pn and RGS spectra}

In order to study the nuclear emission of NGC 1808 in greater detail, we took advantage of the high spectral resolution of the $R G S$ data $(0.35-2.5 \mathrm{keV})$.

Figure 8 compares the combined RGS1 and RGS2 spectrum of NGC 1808 with that of M 82, a proto-typical starburst galaxy. Both spectra were generated with the SAS task rgsfluxer applying the standard extraction techniques. The $R G S$ spectrum of NGC 1808 shows no continuum emission above the noise level, but it does exhibit a number of emission lines. Figure 8 shows that these have wavelengths and relative intensity ratios very similar to the strongest lines identified in M $82($ Read \& Stevens 2002).

We determined the wavelength of the emission lines observed with the RGS by fitting Gaussian profiles to them. The redshift-corrected locations of the fitted Gaussians are less than $0.02 \AA$ from the theoretical value of the lines that we have identified. The fitted line widths are within the range of 0.02-0.04 $\AA$, compatible with the instrumental resolution of the $R G S$. The detected lines are thus identified as: the Ly $\alpha$ emission line from O VIII, transitions of He-like Ne IX ions, and Fe L emission lines from Fe XVII and Fe XVIII. The weaker lines visible in the M 82 spectrum were not detected in NGC 1808, most probably due to the lower S/N. The similarity in the line ratios suggests that there is a common origin of the soft X-ray emission in both galaxies, i.e. thermal emission from a hot, extended gas component, as shown for M 82 (Read $\&$ Stevens 2002). This result confirms the detection of the starburst in NGC 1808 in our data, which dominates the soft X-ray emission.

The RGS spectra of NGC 1808 and M 82 in Fig. 8 show only a qualitative comparison. In particular, in the reduction process of the data, calibration effects affecting the line profile 
were not considered, and therefore the wings of the lines are not properly determined. The most important effect of this issue is that the flux of the lines could be underestimated. The ratio of fluxes of the detected lines can be used to derive physical properties of the gas where the lines originate. In order to perform an accurate study of the properties of the emission lines, we analysed the $R G S$ spectrum, taking the response of the detector into account and therefore avoiding the uncertainties explained before. Using XSPEC v.11.3, we simultaneously analysed the $R G S$ and the $16^{\prime \prime}$ region EPIC-pn spectra. Figure 9 shows that the soft X-ray emission of NGC 1808 can be explained solely by the contribution of emission lines. Consequently, we tried to model the $p n-R G S$ spectra with an absorbed $\left(N_{\mathrm{H}}=1_{-1}^{+17} \times 10^{19} \mathrm{~cm}^{-2}\right)$ power law $\left(\Gamma=1.1_{-0.5}^{+0.6}\right.$ and $A_{\mathrm{pl}}=9.4_{-0.7}^{+0.9} \times 10^{-5}$ photons $/ \mathrm{keV} / \mathrm{cm}^{2} / \mathrm{s}$ at $\left.1 \mathrm{keV}\right)$ which accounts for the hard band emission and a number of lines accounting for the soft band emission. The emission lines were modeled with Gaussian profiles with widths fixed to their instrumental value, both in $p n$ and $R G S$. The wavelengths were also fixed to the theoretical values of the most likely identifications in order to reduce the degrees of freedom. The best fit model consists of 13 emission lines and a power law with a value for $\chi^{2}=307$ for 209 dof. The energy, intensity, and identification of each line are shown in Table 3 and the fit in Fig. 9.

The line ratios provide a diagnostic of the conditions of the gas. In particular, the ratios between lines of iron in different degrees of ionization degrees allow for the best temperature diagnostics. Table 3 gives all the ratios relative to the strongest isolated line, i.e. FeXVII (15.01 $\AA$ ). We therefore use the observed FeXVII line and compare it with FeXVIII and others with higher ionization states to infer the plasma temperature. We use the web version of the ATOMDB v.1.3, http: //cxc . harvard. edu/atomdb and conclude that a collisionally ionized plasma with $k T \sim 0.4 \mathrm{keV}\left(\sim 5 \times 10^{6} \mathrm{~K}\right)$ has a line spectrum compatible with the observed spectrum of NGC 1808. This is not surprising, given our previous results using EPIC data alone.

\subsubsection{EPIC spectrum of the non-nuclear extended region}

In order to investigate the non-nuclear extended emission of NGC 1808, the EPIC-pn spectrum of an annular region was extracted and analysed. The inner and the outer radii, 16".5 $(875 \mathrm{pc})$ and $35^{\prime \prime}(1.9 \mathrm{kpc})$, were chosen to minimize the contribution of the so-called inner region, i.e. $r=16^{\prime \prime}$, and to exclude the CCD edges.

For the spectral fit, we have assumed that the emission in this region is contaminated by the emission from the inner region. Therefore, the fitting model includes a power law with an index fixed to the value obtained in the fit of the inner region spectrum, i.e. $\Gamma=0.79$, and an absorbed mekal component with $N_{\mathrm{H}}$ fixed to $2 \times 10^{21} \mathrm{~cm}^{-2}$ and $k T_{\mathrm{I}}$ fixed to $0.58 \mathrm{keV}$. The normalisations of both components were left free. An excess with respect to the model is observed at low energies. This feature can be well fitted with an extra mekal component with a
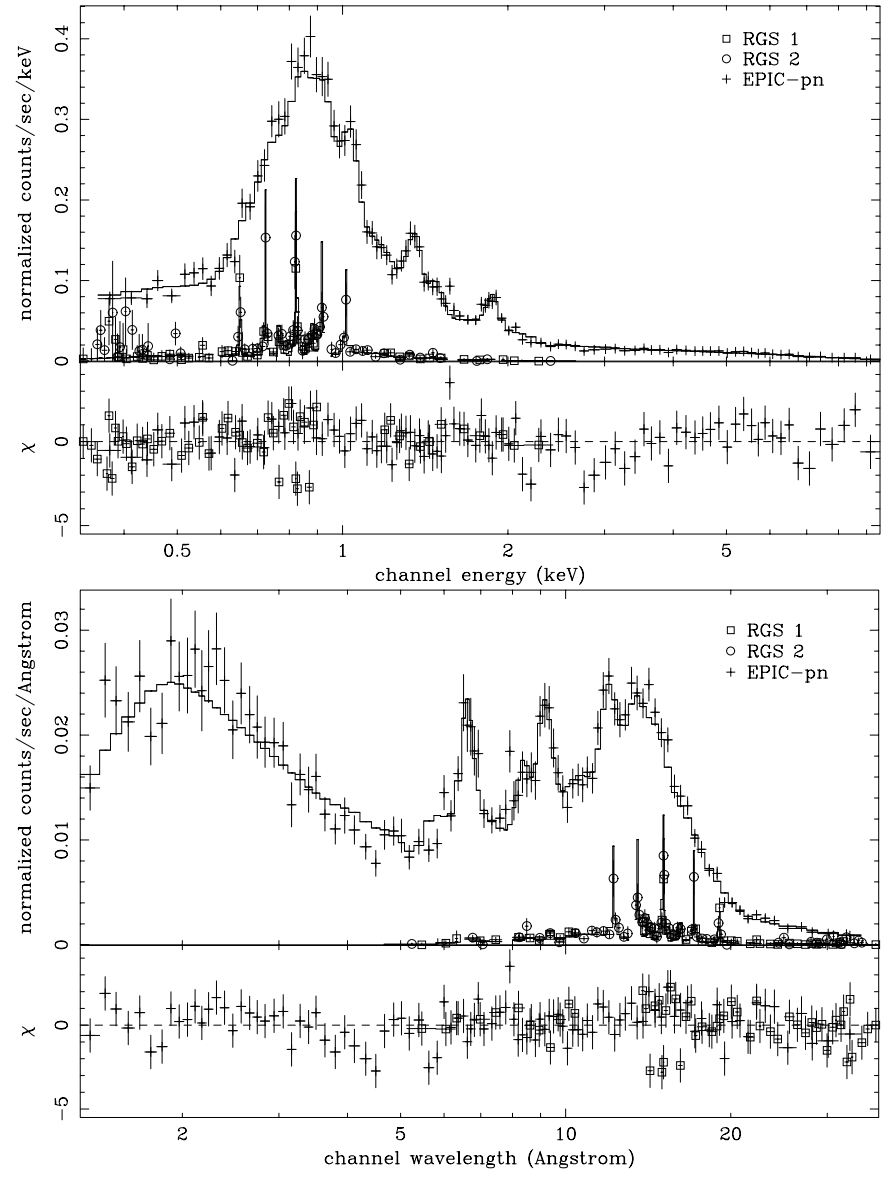

Fig. 9. High-resolution $R G S 1+R G S 2$ and $E P I C$ - $p n$ spectra in both energy (top plot) and wavelength (bottom plot) units, together with the best-fitting model which includes a power law and 13 Gaussian profiles and the EPIC-pn and $R G S-1$ residuals.

temperature $k T_{\text {II }} \simeq 0.1 \mathrm{keV}$. The abundances of $\mathrm{Ne}, \mathrm{Mg}, \mathrm{Si}$, and Fe have been left free in the fits and their values are compatible within the errors with the abundances obtained for the inner region. The values of all parameters and the goodness of the fit can be found in Table 1. Figure 10 shows the data, the best fit model, and the residuals.

Comparison of the inner and annular regions shows that the annular spectrum includes a component not detected in the inner region, the low temperature gas, which can therefore be associated with a non-nuclear, extended emission component.

The results of the analysis confirm our expectation that the spectrum of the annular region is contaminated by some emission from the inner region. This is especially true for the power law, which is fainter by a factor of $\sim 6.5$ compared to that in the inner region, while the hotter thermal gas contribution may be partially due to either extended emission or discrete sources in the annulus, as its normalisation is only a factor of 3.4 weaker than in the inner region. Indeed, the encircled energy function for EPIC-pn predicts that about $20 \%$ of the total energy emitted by the unresolved source will be imaged between $r=16$.' 5 and $r=35^{\prime \prime}$ from the centre of the point spread function. 
Table 3. Properties of the identified lines in the simultaneous analysis of $R G S$ and EPIC-pn data.

\begin{tabular}{|c|c|c|c|c|}
\hline $\begin{array}{l}\text { Energy } \\
\text { keV }\end{array}$ & $\begin{array}{l}\text { Wavelength } \\
\AA\end{array}$ & $\begin{array}{l}\text { Intensity } \\
\mathrm{ph} \mathrm{cm}^{-2} \mathrm{~s}^{-1}\end{array}$ & $I / I_{\mathrm{FeXVII}}$ & Identification \\
\hline 1.99 & 6.23 & $0.02_{-0.02}^{+0.15}$ & 0.01 & SiXIV Ly $\alpha 6.182 \AA$ \\
\hline 1.87 & 6.63 & $0.69_{-0.15}^{+0.17}$ & 0.21 & SiXIII He $\alpha 6.65(6.69) 6.74 \AA$ \\
\hline 1.47 & 8.43 & $0.53_{-0.19}^{+0.11}$ & 0.16 & $\operatorname{MgXII~Ly~} \alpha 8.42 \AA$ \\
\hline 1.34 & 9.25 & $1.09_{-0.19}^{+0.16}$ & 0.33 & $\operatorname{MgXII~He~} \alpha 9.17(9.23) 9.31 \AA$ \\
\hline 1.13 & 10.97 & $3.04_{-0.38}^{+0.41}$ & 0.91 & $\mathrm{FeXVII+FeVIII}$ \\
\hline 1.02 & 12.15 & $2.34_{-0.24}^{+0.26}$ & 0.70 & NeX Ly $\alpha 12.134 \AA+F e X V I I$ \\
\hline 0.917 & 13.52 & $2.88_{-0.29}^{+0.30}$ & 0.86 & NeIX He $\alpha 13.45,13.55,13.70 \AA ̊+F e X V I I$ \\
\hline 0.876 & 14.15 & $1.32_{-0.26}^{+0.26}$ & 0.39 & FeXVIII 14.21,14.37,14.23 ̊̊ \\
\hline 0.823 & 15.06 & $3.34_{-0.29}^{+0.30}$ & 1.00 & FeXVII 15.01,15.26 ̊ \\
\hline 0.769 & 16.12 & $1.32_{-0.26}^{+0.26}$ & 0.40 & FeXVIII 16.00,16.07 Å+OVIII Ly $\beta 16.01 \AA ̊+F e X I X$ \\
\hline 0.724 & 17.12 & $2.49_{-0.32}^{+0.33}$ & 0.75 & FeXVII 16.78,17.05,17.10 ̊ \\
\hline 0.651 & 19.04 & $1.49_{-0.31}^{+0.34}$ & 0.45 & OVIII Ly $\alpha 18.97 \AA ̊$ +CaXVII+CaXVI \\
\hline 0.565 & 21.94 & $0.17_{-0.17}^{+0.42}$ & 0.05 & OVII He $\alpha 21.6,21.8,22.1 \AA$ \\
\hline
\end{tabular}

phabs(zphabs*zpow+13zgauss) $\chi_{v}^{2}=1.47209$ d.o.f.

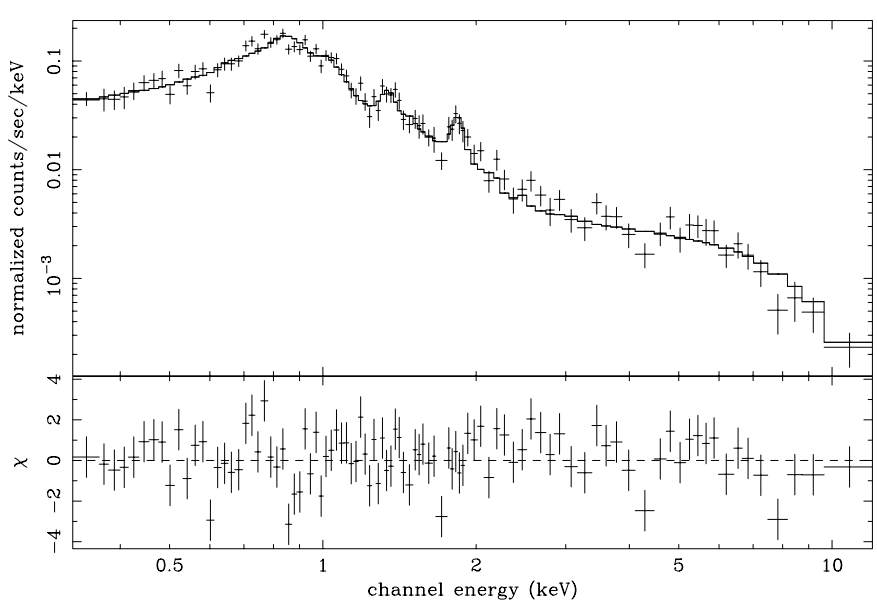

Fig. 10. EPIC-pn spectrum, best fit model, and residuals of the annular region.

\subsubsection{Integrated EPIC spectrum of the total galaxy}

A circular region with $r=62$.'5 $(3.3 \mathrm{kpc})$ was analysed. The extracted EPIC spectrum contains the emission of the whole galaxy and the region coincides with the extraction radius used in the ROSAT analysis (Dahlem et al. 1994; Junkes et al. 1995).

Analogously to the annular region, the components of the best fit model $\left(\chi_{v}^{2}=1.00\right.$ for 222 d.o.f. $)$ are a power law and two absorbed thermal components. The values of the various parameters and the goodness of the fit can be found in Table 1 . Figure 11 shows the data, the best fit model, and the residuals. In the lower panel of Fig. 11, we also show the best fit model and its various additive components in $E f(E)$. The values of the parameters suggest that the emission has the same origin as for the annular region; i.e. the power law and higher temperature component are associated with the nuclear emission, and the soft thermal emission is associated with the non-nuclear extended emission.
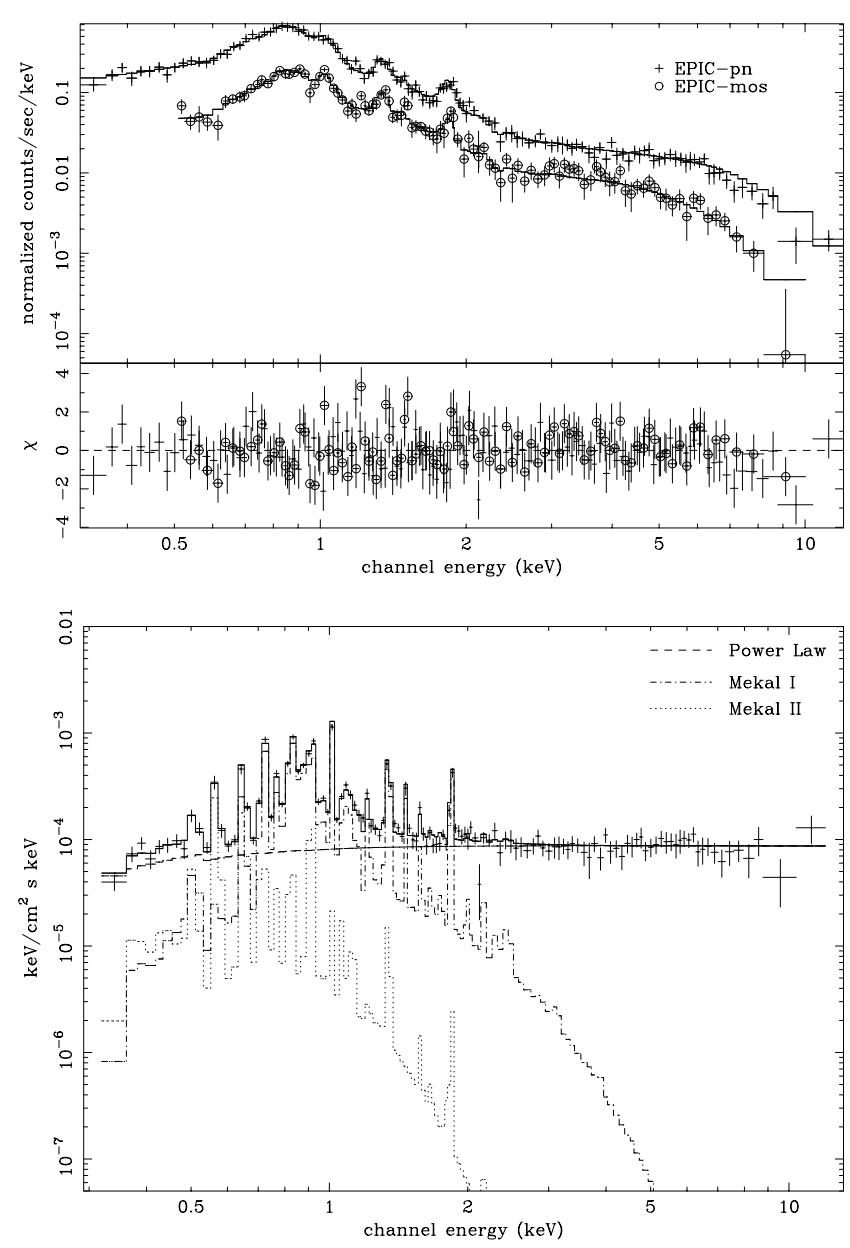

Fig. 11. EPIC spectrum, best fit model, and residuals of the $r=62$ ". 5 $(3.3 \mathrm{kpc})$ region (top plot) and the best fit model, the various additive components, and the EPIC-pn spectrum in $E f(E)$ (bottom plot).

\subsection{X-ray fluxes and luminosities}

Various absorbed X-ray fluxes and unabsorbed luminosities, measured in the three regions of the XMM-Newton analysis and the two regions of the Chandra analysis, are collated in Table 4. 
Table 4. NGC 1808 X-ray absorbed fluxes and unabsorbed luminosities.

\begin{tabular}{|c|c|c|c|c|}
\hline $\begin{array}{l}\text { Region } \\
\text { radius }\end{array}$ & $\begin{array}{l}F_{0.1-2.4 \mathrm{keV}} \\
10^{-13} \mathrm{erg} \mathrm{s}^{-1} \mathrm{~cm}^{-2}\end{array}$ & $\begin{array}{l}F_{2-10 \mathrm{keV}} \\
10^{-13} \mathrm{erg} \mathrm{s}^{-1} \mathrm{~cm}^{-2}\end{array}$ & $\begin{array}{l}L_{0.1-2.4 \mathrm{keV}} \\
10^{39} \mathrm{erg} \mathrm{s}^{-1}\end{array}$ & $\begin{array}{l}L_{2-10 \mathrm{keV}} \\
10^{39} \mathrm{erg} \mathrm{s}^{-1}\end{array}$ \\
\hline $16^{\prime \prime}(850 \mathrm{pc})$ & $5.8_{-1.2}^{+1.1}$ & $11.2 \pm 0.4$ & $18 \pm 4$ & $16.1 \pm 0.6$ \\
\hline Power law & $1.95 \pm 0.17$ & $11.0 \pm 0.4$ & $3.2 \pm 0.3(\sim 20 \%)$ & $15.6 \pm 0.06(\sim 98 \%)$ \\
\hline Thermal & $3.8_{-1.2}^{+1.1}$ & $2.1_{-0.7}^{+0.6}$ & $15 \pm 4(\sim 80 \%)$ & $0.33_{-0.11}^{+0.10}(\sim 2 \%)$ \\
\hline $16^{\prime \prime}-35^{\prime \prime}(875-1900 \mathrm{pc})(\dagger)$ & $1.54_{-0.2}^{+0.16}$ & $1.78_{-0.19}^{+0.17}$ & $8.2_{-1.4}^{+1.0}$ & $2.5 \pm 0.5$ \\
\hline Power law & $0.33_{-0.12}^{+0.3}$ & $1.71_{-0.16}^{+0.17}$ & $0.5 \pm 0.2(\sim 5 \%)$ & $2.4 \pm 0.6(\sim 100 \%)$ \\
\hline Thermal I & $1.1 \pm 0.02$ & $0.075_{-0.002}^{+0.016}$ & $4.3_{-1.3}^{+1.1}(\sim 40 \%)$ & $0.18_{-0.02}^{+0.2}(\sim 5 \%)$ \\
\hline Thermal II & $0.09_{-0.05}^{+0.3}$ & - & $3.4_{-1.2}^{+1.6}(\sim 55 \%)$ & - \\
\hline $62^{\prime \prime} 5(3.3 \mathrm{kpc})(\dagger)$ & $6.8_{-0.7}^{+0.6}$ & $12.1 \pm 0.6$ & $26_{-9}^{+5}$ & $17.2_{-0.6}^{+0.8}$ \\
\hline Power law & $2.1 \pm 1.4$ & $11.9 \pm 0.6$ & $3.3_{-0.3}^{+9}(\sim 10 \%)$ & $16.9_{-0.6}^{+0.7}(\sim 98 \%)$ \\
\hline Thermal I & $4.6_{-0.9}^{+0.6}$ & $0.24_{-0.02}^{+0.07}$ & $15_{-3}^{+7}(\sim 60 \%)$ & $0.38_{-0.08}^{+0.9}(\sim 2 \%)$ \\
\hline Thermal II & $0.18_{-0.11}^{+1.2}$ & - & $7_{-6}^{+7}(\sim 30 \%)$ & - \\
\hline $\mathrm{S} 1(65 \mathrm{pc})$ & $0.63_{-0.10}^{+0.06}$ & $1.0_{-0.5}^{+0.3}$ & $3.9_{-1.0}^{+0.3}$ & $1.5_{-0.9}^{+0.2}$ \\
\hline Power law & $0.22_{-0.06}^{+0.13}$ & $1.0_{-0.6}^{+0.2}$ & $0.39_{-0.03}^{+0.8}(\sim 10 \%)$ & $1.4_{-0.3}^{+0.7}(\sim 95 \%)$ \\
\hline Thermal & $0.41_{-0.06}^{+0.10}$ & $0.045_{-0.002}^{+0.2}$ & $3.5_{-0.8}^{+0.4}(\sim 90 \%)$ & $0.074_{-0.007}^{+0.03}(\sim 5 \%)$ \\
\hline $\mathrm{S} 2(65 \mathrm{pc})$ & $0.27_{-0.18}^{+0.09}$ & $5.4_{-5}^{+0.8}$ & $<6 \times 10^{4}$ & $9.2_{-7}^{+0.8}$ \\
\hline Power law & $0.20_{-0.19}^{+0.12}$ & $5.4_{-5}^{+0.5}$ & $<400(\sim 0 \%)$ & $9.4_{-0.7}^{+0.9}(\sim 100 \%)$ \\
\hline Thermal & $0.07_{-0.03}^{+0.07}$ & $3 \times 10^{-5}$ & $<6 \times 10^{4}(\sim 100 \%)$ & - \\
\hline $16^{\prime \prime}(850 \mathrm{pc})$ Chandra & $5.5_{-0.9}^{+0.4}$ & $9.4_{-0.9}^{+0.6}$ & - & - \\
\hline $16^{\prime \prime}-35^{\prime \prime}(875-1900 \mathrm{pc})$ Chandra & $0.5_{-0.3}^{+0.4}$ & $<0.23$ & - & - \\
\hline 62.'5 (3.3 kpc) Chandra & $6.1_{-1.2}^{+0.4}$ & $9.1_{-1.2}^{+0.4}$ & $26_{-9}^{+2}$ & $22.2_{-1.6}^{+2}$ \\
\hline $62 . .5(3.3 \mathrm{kpc})$ ROSAT & & & $\sim 140$ & - \\
\hline
\end{tabular}

$(\dagger)$ The label Thermal I corresponds to the highest temperature mekal component and the Thermal II to the lowest.

For all cases, the thermal components dominate in the soft $\mathrm{X}$-ray band, while the power law dominates in the $2-10 \mathrm{keV}$ band. The innermost part of the galaxy $\left(r=16^{\prime \prime}=850 \mathrm{pc}\right)$ is responsible for emission in the hard band, while in the soft energy band it contributes only half of the total luminosity of the galaxy $(r=62 \prime \prime 5=3.3 \mathrm{kpc})$.

In order to study the long term variability in NGC 1808, we compared the fluxes observed with XMM-Newton in April 2002 with the ones observed with Chandra eight months later. To allow comparison, the spectra of the nuclear, the annular, and the inner regions were extracted from the Chandra ACIS observation. These regions are equivalent to the ones analysed with XMM-Newton. The spectra of the inner regions were fitted using the best fit model obtained from the XMM-Newton analysis, i.e. a power law plus an absorbed mekal component. The index of the power law, hydrogen column density, and the temperature and abundances of the mekal component of the best fit model $\left(\chi^{2}=170\right.$ for 137 d.o.f. $)$ are compatible with the values obtained with XMM-Newton. Therefore, no spectral variability was detected between both observations. The spectrum of the annular region was also fitted using the best fit model obtained with XMM-Newton. The values of the relevant parameters of the best fit model ( $\chi^{2}=45$ for 36 d.o.f.) are again compatible with the ones derived from the XMM-Newton observation.

In the inner region, the comparison of the XMM-Newton and Chandra values reveals no sign of variability in the soft energy range Chandra flux is only 5\% lower than the
XMM-Newton flux, well within the statistical errors and calibration uncertainties of both instruments. In the hard band, a decrease of $16 \%$ in flux is observed at a $2.5 \sigma$ level (see Table 4).

The Chandra analysis of the two discrete sources detected within the $r=16^{\prime \prime}$ region reveals that source $\mathrm{S} 2$ is responsible for $\sim 50 \%$ of the hard emission from the inner Chandra region, while the combined emission of sources S1 and S2 only explains $\sim 15 \%$ of the observed soft emission in this inner region. It is worth noting that the upper limit of the intrinsic, i.e. absorption-corrected soft X-ray luminosity of S2 obtained with Chandra, is more than three orders of magnitude higher than the luminosity of the whole galaxy. However, considering the uncertainties of this measurement, it is compatible with lower values. Moreover, taking the absorbed fluxes into account, no discrepancy is found, indicating that the intrinsic absorption components are responsible for the high luminosity measured. Although the statistical test shows that the model is acceptable, alternative scenarios cannot be ruled out.

In the annular region, the Chandra flux measurement in the soft band is significantly lower than those of XMM-Newton. However, Chandra is less efficient than XMM-Newton to detect weak extended emission and probably the discrepancy is due to the extended emission not determined by Chandra.

We also measured the fluxes and luminosities with Chandra within an $r=62$ '.5 aperture to compare the XMM-Newton data with Chandra. The Chandra spectrum does not require 
the complexity needed to describe the XMM-Newton spectrum: only a combination of a power law and one mekal component is sufficient. The lowest temperature mekal, $k T \sim 0.1 \mathrm{keV}$, found with XMM-Newton is not detected in the Chandra spectrum due to the small effective area of the instrument at such low energies. The values of the parameters of the two components included in the best-fit model $\left(\chi^{2}=220\right.$ for 200 d.o.f.) are compatible with the XMM-Newton results.

As expected, taking the results for the inner region into account, a mild variability in the hard band was observed between the XMM-Newton and Chandra observations, while no significant variation of the flux was measured in the soft energy range. This variation is dominated by the power law component of the inner region.

In addition to the newly derived luminosity values, Table 4 also lists the luminosity measured with ROSAT (Junkes et al. $1995)$ in this region. The values indicate an important decrease in the luminosity between the ROSAT, XMM-Newton, and Chandra observations. Comparing our results $\left(L_{0.5-10 \mathrm{keV}} \sim\right.$ $3 \times 10^{40} \mathrm{erg} \mathrm{s}^{-1}$ ) for the $r=62$ '. 5 region with the ASCA observations of February 1994 obtained by Awaki et al. 1996 $\left(L_{0.5-10 \mathrm{keV}}=2 \times 10^{40} \mathrm{erg} \mathrm{s}^{-1}\right)$, a small increase in the luminosity is observed. The comparison with the Ginga results (October 1990) indicates a decrease of the luminosity from $L_{2-10 \mathrm{keV}}=5 \times 10^{40} \mathrm{erg} \mathrm{s}^{-1}$ for Ginga to $L_{2-10 \mathrm{keV}}=1.7 \times$ $10^{40} \mathrm{erg} \mathrm{s}^{-1}$ for XMM-Newton. Nevertheless, existing uncertainties in the relative calibration of various X-ray detectors could explain the discrepancy of XMM-Newton and Chandra with the ASCA and Ginga values, but the luminosity measured by ROSAT is 5 times that observed with XMM-Newton and Chandra, too high to be explained by calibration uncertainties.

Short-term variability during the XMM-Newton observation was not detected.

\subsection{OM optical/UV data and H $\alpha$ imagery}

OM UV images of NGC 1808 with the U (350 nm), UVW1 (291 nm) and UVW2 (212 nm) filters were taken in parallel with the X-ray observations. Due to technical problems, part of the UVW1 image was lost. All images are displayed in Fig. 12. The $U$ and UVW2 images show a double-peak structure in the centre of the galaxy. The locations of the peaks in UVW2 image are $\alpha_{1}^{\mathrm{OM}}(\mathrm{J} 2000)=05: 07: 41.97 ; \delta_{1}^{\mathrm{OM}}(\mathrm{J} 2000)=-37: 30: 43.3$ and $\alpha_{2}^{\mathrm{OM}}(\mathrm{J} 2000)=05: 07: 42.62 ; \delta_{2}^{\mathrm{OM}}(\mathrm{J} 2000)=-37: 30: 48.1$ with uncertainties of $\sim 2 "$. Figure $12 \mathrm{c}$ shows the central region of the UVW2 image with the Chandra contours overlaid. The locations of the two point-like sources detected by Chandra (at $\sim 4$ " from the nucleus and aligned in the NW and SE direction) coincide with the positions of the UV peaks observed in the $O M$ image. However, there is no UV emission associated with any of the two bright X-ray sources at the very nucleus of the galaxy.

In Fig. 13 we have overlaid the $O M 212 \mathrm{~nm}$ image of NGC 1808 on the EPIC $0.5-2.0 \mathrm{keV}$ frame from Fig. 1 and on an extremely sensitive $\mathrm{H} \alpha$ image. An RGB composition of the images in optical (H $\alpha)$, UV (OM UVW2) and X-rays (EPIC) is presented in Fig. 14.

The correspondence of UV and $\mathrm{H} \alpha$ emission maxima indicates that the $O M$ data trace the massive stars ionizing the gas in $\mathrm{H}$ II regions in NGC 1808 . The $\mathrm{H} \alpha$ image also shows several knots located in an outer spiral arm. SF is traced in the circumnuclear starburst but also further out in the galaxy disk. Similarly, the soft X-ray emission observed in the same regions is expected to come from hot gas in or near these SF regions. Optical/UV emission also tracks the bar.

\section{Discussion}

ASCA and Ginga observations in the past missed the starburst's soft X-ray emission, favouring an interpretation in terms of an AGN (Awaki et al. 1996; Awaki \& Koyama 1993), as suggested first by Véron-Cetty and Véron (1985). Similarly, the IR emission observed by ISO is dominated by this AGN (Siebenmorgen et al. 2001).

ROSAT, on the other hand, detected the soft emission from the starburst, being less sensitive to the hard emission of an AGN (e.g. Junkes et al. 1995), thus suggesting the predominance of the starburst.

Based on the data presented above, the most likely interpretation is that NGC 1808 hosts both, a circumnuclear starburst and an unresolved nuclear X-ray source. Both in the imaging (Figs. 1, 3 and 4) and spectral (Figs. 6, 7 and 9) domains, XMMNewton and Chandra detect the presence of the starburst and the hard unresolved nuclear source. In the following we discuss the physical origin of the emission of both types of activity: the starburst and the unresolved X-ray source.

\subsection{X-ray emission of the inner region}

\subsubsection{Hard component}

\section{Spectral characteristics}

The slope of the power law fitted to the $16^{\prime \prime}$ region, $\Gamma=$ $0.79_{-0.06}^{+0.07}$, is flatter in comparison to results obtained for radioquiet AGN and LINERs. Works by Georgantopoulos et al. (2002) and Terashima et al. (2002) find values for the power law spectral indices ranging from 1.7 to 2.3 for these objects. However, there are also objects with lower spectral indices (e.g. PKS 2251+113, with $\Gamma=0.95 \pm 0.24$, Reeves \& Turner 2001). Analysis of the Chandra data reveals that this hard emission is dominated by one of the two detected nuclear sources, S2 in Fig. 4, which is harder. In addition, source $\mathrm{S} 2$ is absorbed by a column density of $N_{\mathrm{H}}=3.1_{-0.7}^{+0.8} \times 10^{22} \mathrm{~cm}^{-2}$, compatible with values observed in Compton-thin Seyfert 2 galaxies. This fact suggests that in case one of the nuclear peaks observed with Chandra is associated with an AGN, the most likely possibility is that it is source S2. Interestingly, the best-fit model of the XMM-Newton 16" region spectrum does not require absorption in excess of the Galactic value. However, this XMM-Newton region includes several emitting sources that could blur the presence of an absorbing component in the best fit model. 


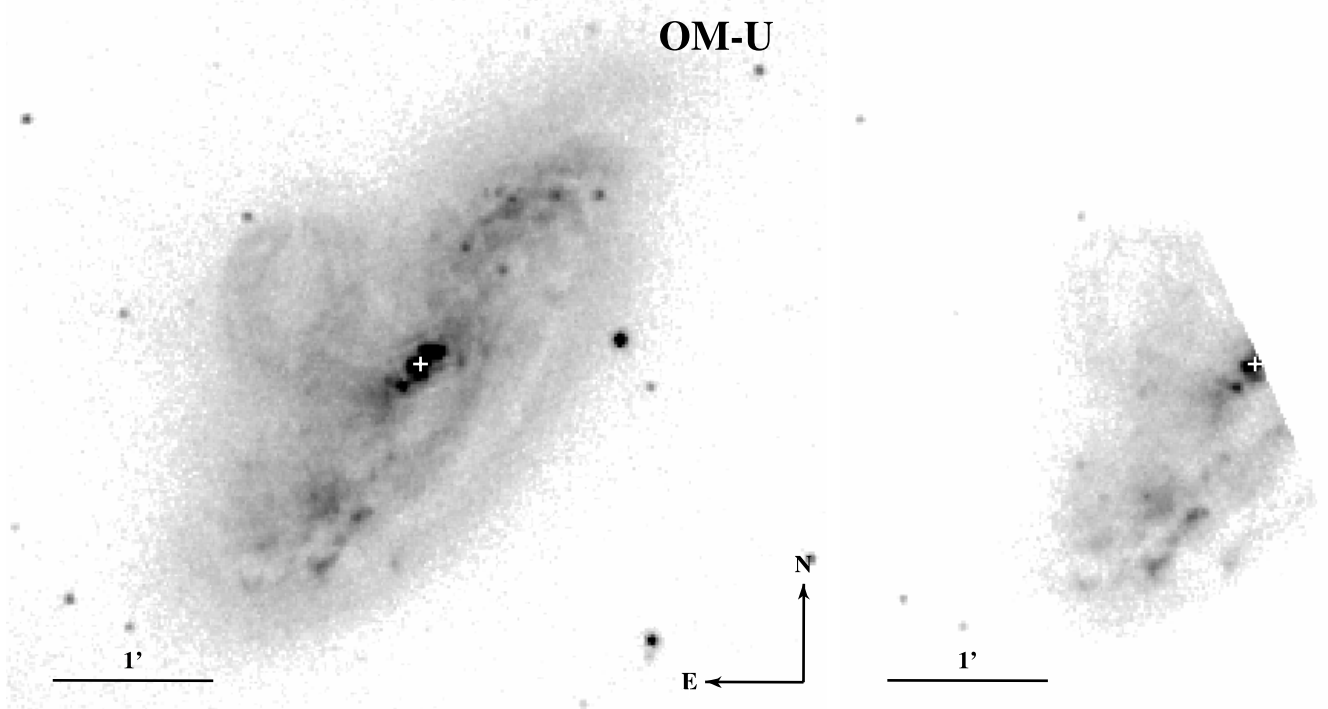

OM-UVW1

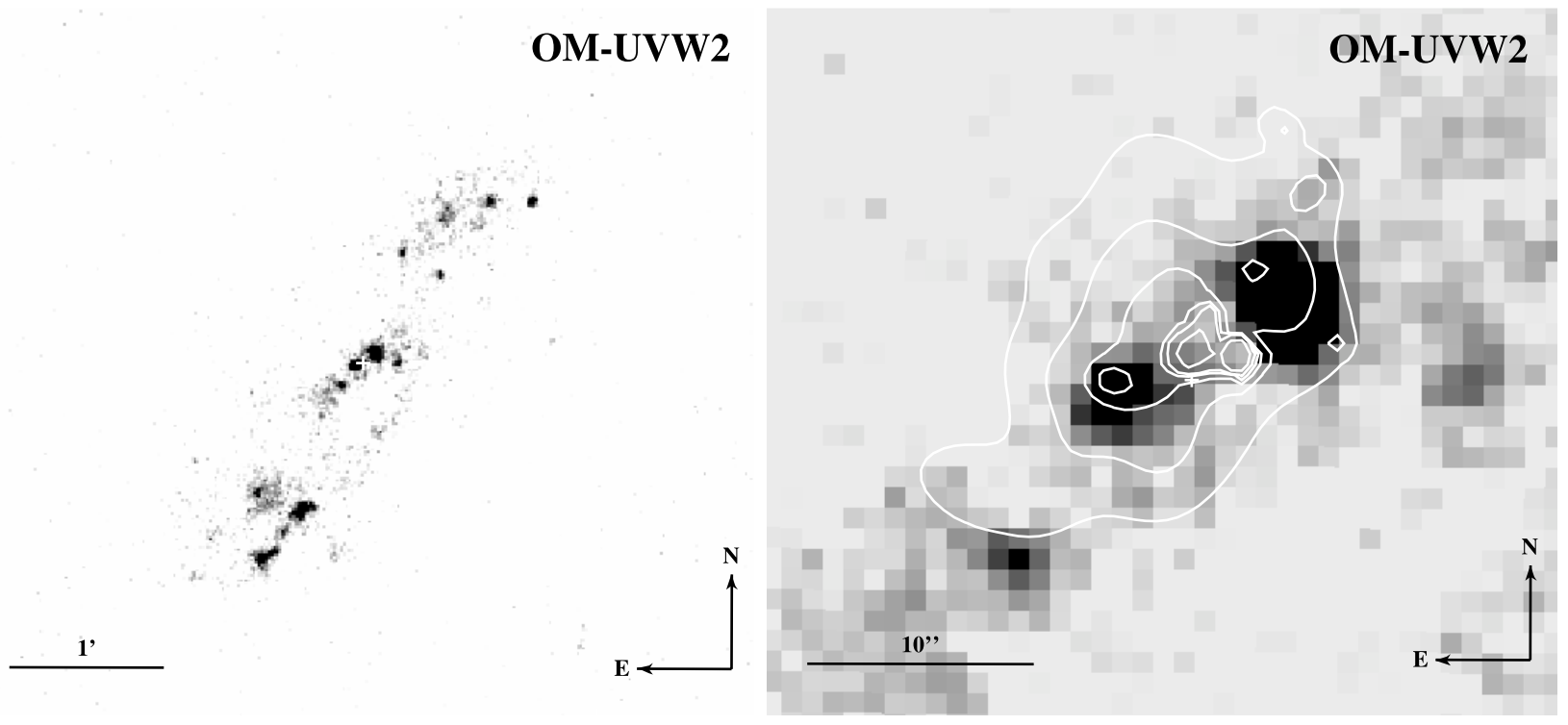

Fig. 12. OM UV images of NGC 1808. From left to right and from top to bottom: $U$ filter ( $350 \mathrm{~nm}), U V W 1$ filter (291 nm), UVW2 (212 nm) images, and the UVW2 central region of NGC 1808 with the overlay of the Chandra contours. The location of the nucleus from 2MASS is marked with a cross. The elliptical ring eastwards of the galaxy in $U$ and $U V W 1$ images is an artefact due to the light scattered within the $O M$ detector. All images are displayed in logarithmic scale with a threshold of $3 \sigma$ of the mean background value.

\section{Luminosity}

The total luminosity of the central region with a radius of $16^{\prime \prime}=850 \mathrm{pc}$ of $\mathrm{NGC} 1808$ is $L_{2-10 \mathrm{keV}}=$ $(1.61 \pm 0.06) \times 10^{40} \mathrm{erg} \mathrm{s}^{-1}$. The luminosity in the same energy band associated with the harder peak with a radius of $1^{\prime \prime} 2=65$ pc observed with Chandra, i.e. source S2, is $L_{2-10 \mathrm{keV}}=0.92_{-0.7}^{+0.08} \times 10^{40} \mathrm{erg} \mathrm{s}^{-1}$, which is $\sim 60 \%$ of the emission of the XMM-Newton inner region. These values are in the range of Low Luminosity AGN (LLAGN) and LINERS (Terashima et al. 2002; Georgantopoulos et al. 2002). Moreover, in both cases the luminosity associated with the power law component is in good agreement with the typical values of low luminosity Seyfert galaxies and LINERs. Terashima et al. (2002) found in a sample of 53 objects of these types observed by ASCA that the luminosity varies within the range $L_{2-10 \mathrm{keV}}=5 \times 10^{39-40} \mathrm{erg} \mathrm{s}^{-1}$. If only
LINERS are considered, the mean luminosity obtained is $L_{2-10 \mathrm{keV}}=7 \times 10^{39} \mathrm{erg} \mathrm{s}^{-1}$ (Ho et al. 2001), which is lower than the one derived for NGC 1808. In the soft band, a sample of LINERS observed with ROSAT (Komossa et al. 1999) shows luminosities in the range of $L_{0.24-2 \mathrm{keV}}=10^{38-41} \mathrm{erg} \mathrm{s}^{-1}$, in agreement with luminosity measured for the NGC 1808.

\section{Variability}

We studied the variability of NGC 1808 . While no shortterm variation of flux was detected during the XMM-Newton observation, medium- and long-term variability has been suggested in both soft and hard bands.

NGC 1808 was observed by XMM-Newton and Chandra with an interval of eight months. The measurements indicate the soft X-ray flux remained constant, taking the uncertainties into account. A decrease of $16 \%$ (at a $2.5 \sigma$ level) in the 

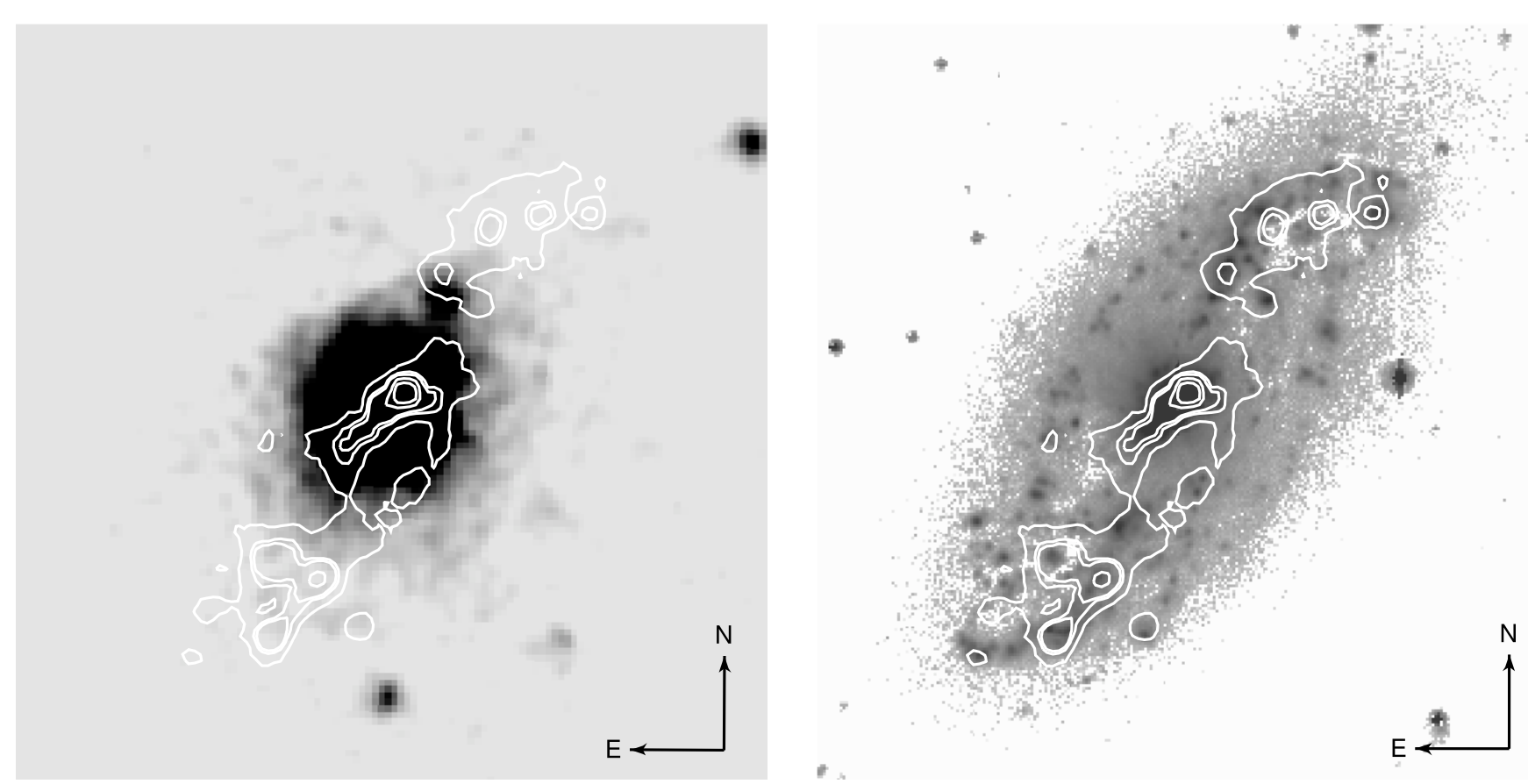

Fig. 13. Overlay of our UVW2 $(212 \mathrm{~nm}$ ) filter $O M$ contours on top the EPIC $0.5-2.0 \mathrm{keV}$ frame (left) and an H $\alpha$ image (right). (Contour levels are $2,7,9,10,15,20,30$, and 80 counts.) All images are displayed in logarithmic scale with a threshold of $3 \sigma$ of the mean background value.

measured flux is observed in the hard X-ray band. In particular, the power law component of the innermost region spectrum of NGC 1808 is responsible for this variability.

The long- and medium-term flux variations measured over the years by various satellites are most probably caused by variability of the nuclear source. In the soft energy band, a significant decrease of a factor of five has been observed when the ROSAT and XMM-Newton (or Chandra) luminosities are compared. It should be noted that the ROSAT spectral fit is particularly uncertain because of the high absorbing column density, which leaves a very narrow effective bandpass for the X-ray emission, from about 0.5 to $2.4 \mathrm{keV}$.

In summary, mild medium-term variability of the hard band luminosity was measured between the XMM-Newton and Chandra observations. This variation can be attributed to an AGN or a ULX. Furthermore, the uncertainties introduced by comparing different satellites does not allow us to firmly conclude that other changes in flux are intrinsically due to the nucleus of NGC 1808.

\section{Ultraluminous $\mathrm{X}$-ray sources}

The study of the hard X-rays emanating from the inner region indicates that the nuclear X-ray emission itself could be due to a LLAGN or a LINER. The observed hard band X-ray luminosity, $L_{2-10 \mathrm{keV}}=(1.61 \pm 0.06) \times 10^{40} \mathrm{erg} \mathrm{s}^{-1}$, is very difficult to explain by standard stellar processes. Nevertheless, non-nuclear point-like sources with X-ray luminosities on the order of $10^{38-41} \mathrm{erg} \mathrm{s}^{-1}$ have been detected in nearby galaxies. An alternative origin to an AGN nucleus of NGC 1808 could be one of the so-called ultraluminous X-ray sources (ULX).

The two central sources $\mathrm{S} 1$ and $\mathrm{S} 2$ were studied in detail in Sect. 3.2.2. The nuclear location from 2MASS is compatible with either of them being the nucleus. The luminosity of source S2 of $L_{2-10 \mathrm{keV}}=9.2_{-7}^{+0.8} \times 10^{39} \mathrm{erg} \mathrm{s}^{-1}$ is compatible with either a LLAGN or a ULX. Strickland et al. (2001) and Roberts et al. (2002) show that the analysis of Chandra ULX spectra favours simple power laws with indices in the range of 1.8-2.9, higher than the value obtained in our analysis of $0.79_{-0.06}^{+0.07}$. Interestingly, one of the compact radio sources reported by Saika et al. (1990) coincides with the X-ray pointlike source $\mathrm{S} 1$.

Although more data analysis is necessary to reach any firm conclusion, this finding leaves open the possibility that the nucleus of NGC 1808 could be a ULX.

\subsubsection{Soft component}

\section{Emission distribution}

The soft X-ray emission of the inner region of NGC 1808 is extended (see e.g. Fig. 1). The elongated emission is detected at energies up to $4.5 \mathrm{keV}$. Moreover, the Chandra images clearly show the simultaneous presence of a double emission peak and diffuse, or at least unresolved, X-ray emission around the two peaks. The extended emission is likely to have been created by star formation processes. Our XMM-Newton data permit a clear spectral distinction between the emission associated with the starburst and the additional nuclear sources in the $r=16^{\prime \prime}(850 \mathrm{pc}$ ) region, see Fig. 6. The starburst, associated with the mekal component in the spectral fit, dominates the soft energy band (below $1 \mathrm{keV}$ ), see Tables 1 and 4 . The starburst also shows up in the form of radio continuum and IR knots (Saikia et al. 1990; Kotilainen et al. 1996), bright $\mathrm{H} \alpha$ emission, and bright circumnuclear UV continuum from massive stars, as displayed in Fig. 13. In particular, the position of the 2MASS nucleus coincides perfectly with one of the radio compact sources detected by Saikia et al. (1990), explained as 


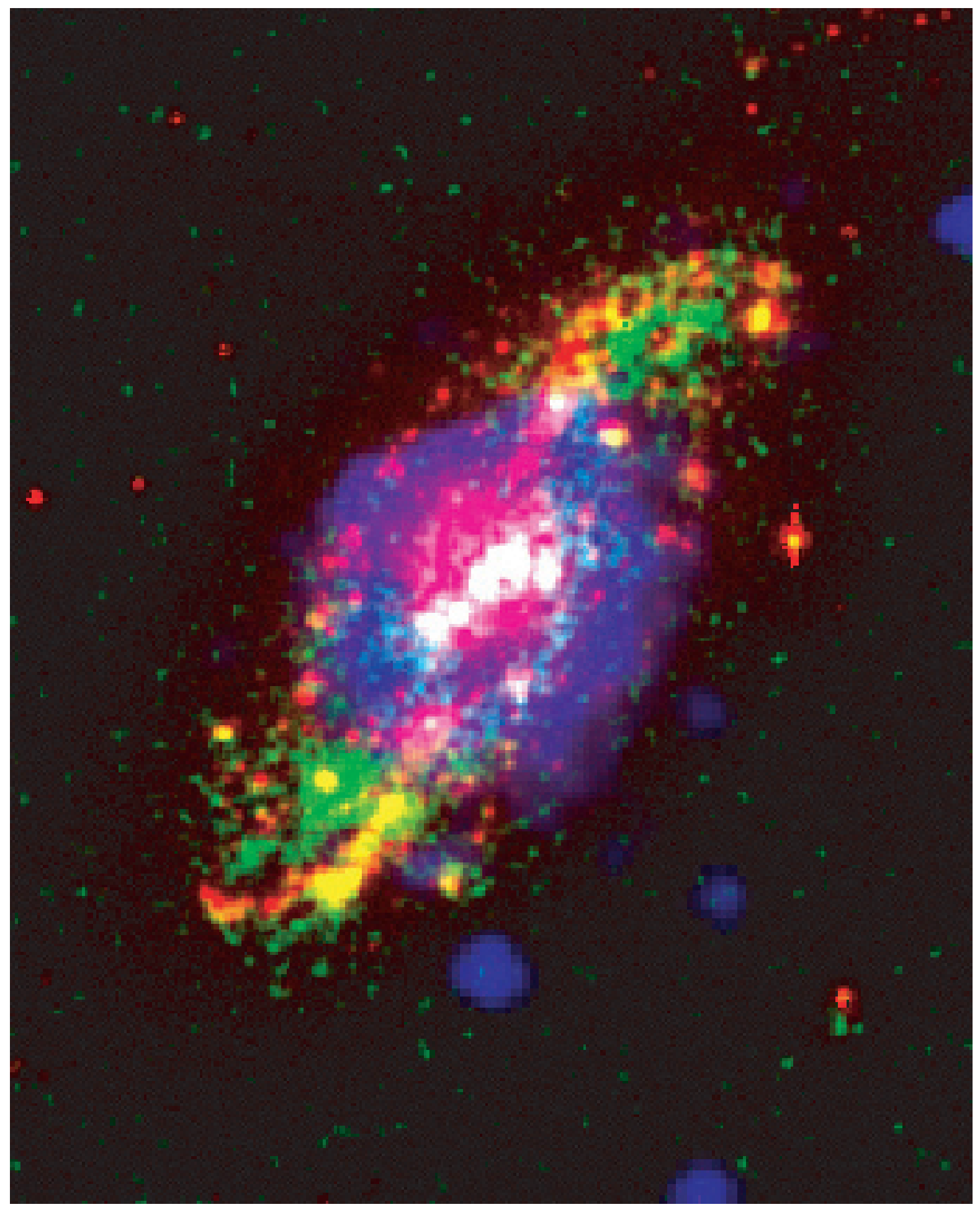

Fig. 14. RGB combination of $\mathrm{H} \alpha$ image (in red), UVW2 (in green), and EPIC (in blue). North is up and East is left. The dimensions of the image are $5^{\prime} \times 3{ }^{\prime} .5$.

SNR or complexes of unresolved SNR. The nucleus is prominent in X-rays and in the optical regime (see Figs. 1 and 12a).

However, while the OM UVW2 image shows that the UV emission close to the position of the nucleus is very weak (see Fig. 12c). These facts suggest that the nucleus is highly absorbed. The spectral analysis of source S2 shows a high value of the equivalent hydrogen column, $N_{\mathrm{H}} \sim 10^{22} \mathrm{~cm}^{-2}$, which can explain this absence of UV and soft X-ray emission associated with the hard X-ray peak.

\section{Plasma temperature}

The best fit to the EPIC data contains a thermal plasma component with a temperature of $k T=0.580_{-0.016}^{+0.019} \mathrm{keV}$. This corresponds roughly to the "medium" component identified in 
the spectra of other starburst galaxies (Dahlem et al. 1998; see also Weaver et al. 2000; Dahlem et al. 2000). It is consistent with $0.5 \pm 0.2 \mathrm{keV}$ (Junkes et al. 1995), which was measured from ROSAT data, based only on about 600 photons. It is interesting to note that the surprisingly high Hydrogen column density, on the order of $8 \times 10^{21} \mathrm{~cm}^{-2}$, in the best-fitting model to the ROSAT data is also corroborated by our results.

\section{X-ray luminosity and SFR}

The luminosity of the starburst component, which we identify with the thermal spectral components, is dominated by the emission in the soft energy band. The observed X-ray luminosity, $L_{0.2-4 \mathrm{keV}} \sim 1.3 \times 10^{40} \mathrm{erg} \mathrm{s}^{-1}$, which represents $\sim 70 \%$ of the emission associated with the starburst component of the whole galaxy, leads to a far-infrared-to-X-ray luminosity ratio of $\log \left(L_{\mathrm{X}} / L_{\mathrm{FIR}}\right)=-3.8^{2}$. This value is consistent with those obtained by Heckman et al. (1990) for a sample of six starburst galaxies, which are in the range between -3.7 and -4.3 , and also with the results in Mas-Hesse et al. (1995), who obtained a mean value of -3.33 for a sample of starforming galaxies.

Ranalli et al. (2003) deduced that the X-ray luminosity is a tracer of SFR, based on a sample of nearby galaxies. As was pointed out by Persic et al. (2004b), the luminosity in the 2$10 \mathrm{keV}$ band is not a precise SFR indicator, due to the contamination by low-mass X-ray binaries (LMXB). These objects are bright in the $\mathrm{X}$-ray band over long timescales, $t_{\mathrm{X}} \sim 10^{7} \mathrm{yr}$, and therefore do not trace the instantaneous SFR. For this reason, we use the soft X-ray luminosity as a tracer of the SRF, with the $S F R-L_{0.5-2 \mathrm{keV}}$ relation in Ranalli et al. (2003):

$S F R_{\mathrm{X}}\left(M_{\odot} \mathrm{yr}^{-1}\right)=2.2 \times 10^{-40} L_{0.5-2 \mathrm{keV}}$

The $L_{0.5-2 \mathrm{keV}}$ associated with the starburst in the $r=16^{\prime \prime}(850 \mathrm{pc})$ region, as derived from the mekal component contribution, is on the order of $L_{0.5-2 \mathrm{keV}}^{S B} \sim 1 \times 10^{40} \mathrm{erg} \mathrm{s}^{-1}$ and the $S F R_{\mathrm{X}}$, therefore, is $\sim 2.5 M_{\odot} \mathrm{yr}^{-1}$.

\section{Metallicities}

We detect several key emission lines, which for the first time provide us with a good measure of the abundances of some elements (Sect. 3.2.1). The RGS spectrum shows a very weak continuum emission with several emission lines. The values obtained indicate slightly super-solar metallicities for $\mathrm{Ne}$, $\mathrm{Mg}$, and $\mathrm{Si}$ and solar or sub-solar abundance for $\mathrm{Fe}$, which is consistent with the results by Dahlem et al. (1998) and Weaver et al. (2000).

In this context the absence of a thermal $\mathrm{Fe} \mathrm{K} \alpha$ line at $\sim 6.5 \mathrm{keV}$ energy is noteworthy. From our data in the annular region, we derive a 3- $\sigma$ upper limit on the equivalent width of $170 \mathrm{eV}$ and on the flux of $4 \times 10^{-7}$ photons $\mathrm{cm}^{-2} \mathrm{~s}^{-1}$. This result is compatible with any possible iron line emission entirely due to the mekal component. We have used ATOMDB v.1.3 to derive the expected flux of the emission lines for a gas with temperature and normalisation as obtained from the single mekal component fitted to the annular spectrum, i.e.

${ }^{2} L_{\mathrm{FIR}}=2 \pi D^{2} \mathrm{FIR}=9.3 \times 10^{43} \mathrm{erg} \mathrm{s}^{-1}$ (Dahlem et al. 1992), where FIR $=1.26\left(2.58 f_{60}+f_{100}\right), f_{60}$ being the IRAS $60 \mu \mathrm{m}$ flux density and $f_{100}$ the $100 \mu \mathrm{m}$ flux density. $k T=0.53_{-0.03}^{+0.05} \mathrm{keV}$ and normalisation $A_{\text {mekal }}=2.2 \times 10^{-4}$. The total flux of the most prominent iron lines (FeXXI-6.505 keV and FeXXII-6.504 \& $6.57 \mathrm{keV}$ ) is $\sim 2 \times 10^{-4}$ photons $\mathrm{cm}^{-2} \mathrm{~s}^{-1}$ (with an emissivity of $\sim 5 \times 10^{-22}$ photons $\mathrm{cm}^{3} \mathrm{~s}^{-1}$ ), consistent with the upper limit measured.

\section{2. $X$-ray emission outside the central region}

In Sect. 3.2, we show the results of the spectral analysis of the $875 \mathrm{pc}-1.9 \mathrm{kpc}$ annular region. The best fit model indicates that, apart from the contribution of the nuclear emission, the annular region includes an extra thermal component which can be explained by a mekal model with $k T=0.10_{-0.01}^{+0.02} \mathrm{keV}$. The analysis of the total galaxy $\mathrm{X}$-ray emission also indicates the presence of a soft thermal model with a similar temperature, $k T=0.11_{-0.02}^{+0.03} \mathrm{keV}$.

Although weak, the contribution in the soft band of this component $\left(L_{0.1-2.4 \mathrm{keV}}=7.7 \times 10^{39} \mathrm{erg} \mathrm{s}^{-1}\right)$ to the soft X-ray luminosity of the whole galaxy is significant, representing around $30 \%$ of the total soft X-ray emission of NGC 1808. In the hard band, the contribution of this low temperature thermal component is negligible. Using Eq. (1) (see Sect. 4.1.2 for further details), the observed X-ray luminosity associated with both mekal components of the annular region, $L_{0.5-2 \mathrm{keV}} \sim$ $4 \times 10^{39}$, can be translated into a $S F R$ of $\sim 1 M_{\odot} \mathrm{yr}^{-1}$.

Far-infrared emission is also a good tracer of the star formation; using the relationship by Kennicutt et al. (1998), we derive for NGC 1808:

$$
S F R_{\mathrm{FIR}}\left(M_{\odot} \mathrm{yr}^{-1}\right)=\frac{1}{e} 4.5 \times 10^{-44} L_{\mathrm{FIR}}
$$

where $e$ is the fraction of UV/optical flux emitted by the stars and absorbed by the dust and remitted in the IR, which is close to unity even for low values of reddening (Mas-Hesse \& Kunth 1991). Assuming that the whole FIR emission is due to the starburst, we obtain $\mathrm{SFR}_{\mathrm{FIR}} \sim 4 M_{\odot} \mathrm{yr}^{-1}$. This result is consistent with the one obtained through the X-ray emission, combining the values derived from the nuclear region $\left(S F R_{\mathrm{X}} \sim 2.5 M_{\odot} \mathrm{yr}^{-1}\right.$, see Sect. 4.1.2) and from the annular region $\left(S F R_{\mathrm{X}} \sim 1 M_{\odot} \mathrm{yr}^{-1}\right)$. It suggests that the contribution of the AGN to the FIR emission is small.

The second, soft mekal component detected in both the annular and the $r=3.3 \mathrm{kpc}$ region is most likely thermal emission associated with the halo. Such a thermal component $(k T=0.2-0.3 \mathrm{keV})$ is found in all starburst galaxies in the sample by Dahlem et al. (1998) and Weaver et al. (2000) and also by us in other starburst galaxies observed with XMM-Newton (e.g. Dahlem et al. 2003; Ehle \& Dahlem 2004). The presence of both, the prominent vertical dust filaments in NGC 1808 and the gaseous outflow inferred by the kinematics of $\mathrm{H}$ I gas (Koribalski et al. 1993), suggested that this component would most likely be present in NGC 1808 .

If the diffuse X-ray emission in the soft energy band is assumed to be due to thermal cooling of a hot gas in collisional ionization equilibrium ${ }^{3}$, it is possible to estimate the gas density $n_{\mathrm{e}}$, mass $m_{\text {gas }}$, and cooling time $\tau$ of that plasma. To this

\footnotetext{
${ }^{3}$ See, however, the discussion in the validity of this assumption in Breitschwerdt \& Schmutzler (1999).
} 
Table 5. Parameters for the hot gas component in the extra-nuclear starburst region of NGC 1808.

\begin{tabular}{ccc}
\hline \hline $\begin{array}{c}n_{\mathrm{e}} \\
\left(\mathrm{cm}^{-3}\right)\end{array}$ & $\begin{array}{c}m_{\mathrm{gas}} \\
\left(M_{\odot}\right)\end{array}$ & $\begin{array}{c}\tau \\
(\mathrm{yr})\end{array}$ \\
\hline $3.0 \times 10^{-2} / \sqrt{\eta}$ & $6.3 \times 10^{6} \sqrt{\eta}$ & $6.3 \times 10^{7} \sqrt{\eta}$ \\
\hline
\end{tabular}

end we make use of a formula given by Nulsen et al. (1984), i.e. $L_{\mathrm{x}}(\mathrm{soft})=0.812 \Lambda(T) n_{\mathrm{e}}^{2} V \eta$. The unknown filling factor $\eta$ allows for some clumpiness of the gas filling the emitting volume V, and was found in simulations (Avillez \& Breitschwerdt 2004 , and references therein) to be in the range of 0.2-0.4, only modestly increasing with higher $\mathrm{SN}$ rate.

As explained in Sect. 3.2.2, the spectrum of the non-nuclear extended X-ray emission is complex and a best fit was obtained with a model consisting of a foreground absorbed power law plus (at least) two internally absorbed mekal components, corroborating the fact that the hot gas is a multi-temperature mixture. Nevertheless for the purpose of estimating hot gas parameters, we fitted this emission component with a single "characteristic" temperature that was found to be $0.53_{-0.03}^{+0.05} \mathrm{keV}$. For such a gas temperature of the starburst component $(\sim 6.2 \times$ $10^{6} \mathrm{~K}$ ) Raymond et al. (1976) give a cooling coefficient $\Lambda(T)$ of $\sim 5 \times 10^{-23} \mathrm{erg} \mathrm{cm}^{3} \mathrm{~s}^{-1}$. For the unabsorbed luminosity of the hot gas component we make use of the total (Thermal I + II) $L_{0.1-2.4 \mathrm{keV}}=7.7 \times 10^{39} \mathrm{erg} \mathrm{s}^{-1}$ (see Table 4). Assuming for the emitting volume a torus geometry with an inner radius of $875 \mathrm{pc}$ and outer radius of $1.9 \mathrm{kpc}$, the calculated gas parameters are presented in Table 5.

The mass of the detected hot gas is only $\$ 0.5 \%$ of the total gas mass ${ }^{4}$. The cooling time, $\tau$, is comparable to typical duty cycles of starbursts, which are estimated to be on the order of a few times $\sim 10^{7} \mathrm{yr}$ (Rieke et al. 1988).

No significant X-ray emission was found, at the sensitivity of our current data, in the intergalactic space around NGC 1808. Similarly, no $\mathrm{H}$ I and $\mathrm{H} \alpha$ emission was found by us (Dahlem et al. 2001).

\section{Summary and conclusions}

The work presented in this paper shows evidence of the presence of starburst activity and a hard unresolved source within the inner $r=850 \mathrm{pc}$ region in NGC 1808. Analysis of the optical-to-X-ray emission proves the co-existence of thermal diffuse plasma, non nuclear point-like sources and, of a LLAGN or a ULX.

The XMM-Newton EPIC images show extended X-ray emission for energies below $4.5 \mathrm{keV}$. The elongated shape observed in the X-ray band follows the same orientation as the $\mathrm{H} \alpha$ and optical-UV emission, suggesting that SF contributes significantly to the soft X-ray emission. At least four non-nuclear point-like sources were detected in the Chandra ACIS image of the inner $250 \mathrm{pc}$ of NGC 1808. The high spatial resolution Chandra image allows the detection of a double-peak nuclear

\footnotetext{
4 The total gas mass calculated using $\mathrm{H}_{\mathrm{I}}$ observations is $>1.8 \times$ $10^{9} M_{\odot}($ Dahlem et al. 2001).
}

structure. The locations of both sources are compatible with the position of the nucleus as derived from 2MASS. The spectral characteristics of these sources show that one is dominated by hot gas emission, indicating starburst activity, while the other is a highly absorbed hard X-ray source.

The spectral analysis of the XMM-Newton data completes the scenario outlined by X-ray imaging. Comparing EPIC-pn spectra of several regions in the central part of NGC 1808, it is inferred that the bulk of the emission originates from the nucleus, although in the soft X-ray band the contribution of the circumnuclear regions is not negligible.

The X-ray EPIC spectrum of the inner nuclear region $(r=$ $16^{\prime \prime}, 850 \mathrm{pc}$ ) is explained by a power law, $\Gamma=0.79_{-0.06}^{+0.07}$, accounting for the hard X-ray emission and associated with an unresolved point-like source. The absorption measured in the Chandra spectral analysis of source S2 is compatible with typical values of Compton-thin Seyfert 2 galaxies. However, this absorbing material does not show up in the XMM-Newton spectrum, probably because it includes contributions from different regions, making it difficult to separate absorption and soft emission components.

In order to explain the soft X-ray emission of the inner region, it is necessary to include emission due to a diffuse plasma with $k T=0.580_{-0.016}^{+0.019} \mathrm{keV}$. The data obtained with $R G S$ exhibit emission lines similar in wavelength and relative intensity ratios to the ones found for the prototypical starburst galaxy M 82. The temperature, the luminosity, and the various ratios of emission lines are consistent with being originated in a collisionally ionized plasma associated with SF regions, which dominates the total emission spectrum including the continuum in the soft X-ray regime.

The luminosities measured with XMM-Newton for the $r=$ $850 \mathrm{pc}$ inner region $\left(L_{2-10 \mathrm{keV}}=(1.61 \pm 0.06) \times 10^{40} \mathrm{erg} \mathrm{s}^{-1}\right)$ and with Chandra for source S2 $\left(L_{2-10 \mathrm{keV}}=0.92_{-0.7}^{+0.08} \times\right.$ $10^{40} \mathrm{erg} \mathrm{s}^{-1}$ ) are both in the range of LLAGNs but are also compatible with the values of ULX sources. A decrease in the $2-10 \mathrm{keV}$ flux on the order of $15 \%$ in an interval of eight months was measured when comparing XMM-Newton and Chandra observations, while no change in flux was detected in the soft energy band. Both the luminosities and the variability detected are compatible with the unresolved source being a LLAGN or a ULX.

Halo emission of NGC 1808 could also be detected: a softer thermal component, $k T \sim 0.1 \mathrm{keV}$, with $L_{0.1-2.4 \mathrm{keV}} \sim 7 \times$ $10^{39} \mathrm{erg} \mathrm{s}^{-1}(\sim 30 \%$ of the total soft X-ray emission) is necessary to explain the spectrum at distances larger than $875 \mathrm{pc}$ from the centre.

The multiwavelength analysis of the nuclear region of NGC 1808 performed in this work allows us to detect and for the first time to unambiguously disentangle the contributions of an unresolved nuclear X-ray source and the starburst regions within the $r=850 \mathrm{pc}$ region. Further analysis of better quality data is required to determine the exact nature of the unresolved nuclear source.

Acknowledgements. We specially thank N. Stuhrmann for reducing the $\mathrm{H} \alpha$ data. E.J.B., M.S.L. and J.M.M.H. have been supported by Spanish MEC under grant AYA2001-3939-C03-02 


\section{References}

Arnaud, K. A. 1996, Astronomical Data Analyis and Systems, ASP Conf. Ser., 101, 17

Avillez, M. A., de, \& Breitschwerdt, D. 2004, A\&A, 425, 899

Awaki, H., \& Koyama, K. 1993, Adv. Space Res., 13, 221

Awaki, H., Ueno, S., Koyama, K., et al. 1996, PASJ, 48, 409

Breitschwerdt, D., \& Schmutzler, T. 1999, A\&A, 347, 650

Colina, L., Gonzalez Delgado, R., Mas-Hesse, J. M., \& Leitherer, C. 2002, ApJ, 579, 545

Dahlem, M., Aalto, S., Klein, U., et al. 1990, A\&A, 240, 237

Dahlem, M., Bomans, D. J., \& Will, J. 1994, ApJ, 432, 590

Dahlem, M., Weaver, K. A., \& Heckman, T. M. 1998, ApJS, 118, 401

Dahlem, M., Parmar, A., Oosterbroek, T., et al. 2000, ApJ, 538, 555

Dahlem, M., Ehle, M., \& Ryder, S. D. 2001, A\&A, 373, 485

Dahlem, M., Ehle, M., Jansen, F., et al. 2003, A\&A, 403, 547

den Herder, J. W., Brinkman, A. C., Kahn, S. M., et al. 2001, A\&A, $365, \mathrm{~L} 7$

Dickey, J. M., \& Lockman, F. J. 1990, ARA\&A, 28, 215

Ehle, M., \& Dahlem, M. 2004, MmSAI, 75, 515

Gabriel, C., et al. 2004, ASP Conf. Ser., 314, Astronomical Data Analysis Software and Systems (ADASS) XIII, 13, 759

Georgantopoulos, I., Panessa, F., Akylas, A., et al. 2002, A\&A, 386, 60

Heckman, T. M., Armus, L., \& Miley, G. K. 1990, APJS, 74, 833

Ho, L. C., Feigelson, E. D., Townsley, L. K., et al. 2001, ApJ, 549, L51

Jansen, F., Lumb, D., Altieri, B., et al. 2001, A\&A, 365, L1

Jiménez-Bailón, E., Santos-Lleó, M., Mas-Hesse, J. M., et al. 2003, ApJ, 593, 127

Junkes, N., Zinnecker, H., Hensler, et al. 1995, A\&A, 294, 8

Kennicutt, R. C. 1998, ARA\&A, 36, 189

Kirsch, M., et al. 2005, EPIC status of calibration and data analysis, XMM-SOC-CAL-TN-0018,

http://xmm.vilspa.esa.es/external/xmm_sw_cal/ calib/index.shtml

Komossa, S., Böhringer, H., \& Huchra, J. P. 1999, A\&A, 349, 88

Koribalski, B., Dahlem, M., Mebold, U., \& Brinks, E. 1993, A\&A, 268,14

Kotilainen, J. K., Forbes, D. A., Moorwood, A. F. M., van der Werf, P. P., \& Ward, M. J. 1996, A\&A, 313, 771
Mas-Hesse, J. M., \& Kunth, D. 1991, A\&AS, 88, 399

Mas-Hesse, J. M., Rodríguez-Pascual, P. M., Sanz Fernandez de Cordoba, L., et al. 1995, A\&A, 298, 22

Mason, K. O., Breeveld, A., Much, R., et al. 2001, A\&A, 365, L36

Mewe, R., Lemen, J. R., \& van den Oord, G. H. J. 1986, A\&AS, 65, 511

Nulsen, P. E. J., Stewart, G. C., \& Fabian, A. C. 1984, MNRAS, 208, 185

Persic, M., Cappi, M., Rephaeli, et al. 2004a, A\&A, 427, 35

Persic, M., Rephaeli, Y., Braito, V., et al. 2004b, A\&A, 419, 849

Piconcelli, E., Jimenez-Bailón, E., Guainazzi, M., et al. 2004, MNRAS, 351, 161

Ranalli, P., Comastri, A., \& Setti, G. 2003, A\&A, 399, 39

Raymond, J. C., Cox, D. P., \& Smith, B. W. 1976, ApJ, 204, 290

Read, A. M., \& Stevens, I. R. 2002, MNRAS, 335, L36

Reeves, J. N., \& Turner, M. J. L. 2000, MNRAS, 316, 234

Rieke, G. H., Lebofsky, M. J., \& Walker, C. E. 1988, ApJ, 325, 679

Roberts, T. P., Warwick, R. S., Ward, M. J., \& Murray, S. S. 2002 MNRAS, 337, 677

Terashima, Y., Iyomoto, N., Ho, L. C., \& Ptak, A. F. 2002, ApJS, 139, 1

Sandage, A., \& Tammann, G. A. 1987, A Revised Shapley-Ames Catalog of Bright Galaxies (Washington: Carnegie Institution), 2nd ed.

Saikia, D. J., Unger, S. W., Pedlar, et al. 1990, MNRAS, 245, 397

Scarrott, S. M., Draper, P. W., Stockdale, et al. 1993, MNRAS, 264, L7

Siebenmorgen, R., Krügel, E., \& Laureijs, R. J. 2001, A\&A, 377, 735

Sérsic, J. L., \& Pastoriza, M. 1965, PASP, 77, 287

Strickland, D. K., Colbert, E. J. M., Heckman, T. M., et al. 2001, ApJ, 560,707

Strüder, L., Briel, U., Dennerl, K., et al. 2001, A\&A, 365, L18

Turner, M. J. L., Abbey, A., Arnaud, M., et al. 2001, A\&A, 365, L27

Véron-Cetty, M. P., \& Véron, P. 1985, A\&A, 145, 425

Watson, M. G., Auguères, J. L., Ballet, J., et al. 2001, A\&A, 365, L51

Weaver, K. A., Heckman, T. M., \& Dahlem, M. 2000, ApJ, 534, 684

Zezas, A., Fabbiano, G., Prestwich, A., Murray, S., \& Ward, M. 2001, The Central Kiloparsec of Starbursts and AGN: The La Palma Connection, ASP Conf. Ser., 249, 425 